\title{
Regularity for a Nonlinear Discontinuous Subelliptic System with Drift on the Heisenberg Group
}

\author{
Junli Zhang $\mathbb{D}^{1}$ and Jialin Wang $\mathbb{D}^{2}$ \\ ${ }^{1}$ School of Mathematics and Statistics, Northwestern Polytechnical University, Xi'an, Shaanxi 710129, China \\ ${ }^{2}$ School of Mathematics and Computer Science, Gannan Normal University, Ganzhou, Jiangxi 341000, China \\ Correspondence should be addressed to Junli Zhang; jlzhang@mail.nwpu.edu.cn
}

Received 2 September 2021; Accepted 6 October 2021; Published 24 January 2022

Academic Editor: Pietro d Avenia

Copyright (c) 2022 Junli Zhang and Jialin Wang. This is an open access article distributed under the Creative Commons Attribution License, which permits unrestricted use, distribution, and reproduction in any medium, provided the original work is properly cited.

In this paper, we prove the partial Hölder regularity of weak solutions and the partial Morrey regularity to horizontal gradients of weak solutions to a nonlinear discontinuous subelliptic system with drift on the Heisenberg group by the $A$-harmonic approximation, where the coefficients in the nonlinear subelliptic system are discontinuous and satisfy the VMO condition for $x$, ellipticity and growth condition with the growth index $1<p<2$ for the Heisenberg gradient variable, and the nonhomogeneous terms satisfy the controllable growth condition and the natural growth condition, respectively.

\section{Introduction}

Kohn in [1] proved $L^{2}$ estimates for the operator

$$
L u=\sum_{j=1}^{k} X_{j}^{2} u+X_{0} u+c u
$$

constructed by Hörmander's vector fields $\left\{X_{1}, X_{2}, \cdots, X_{q}\right.$, $\left.X_{0}\right\}$ (see [2]) based on the energy estimate and a subelliptic estimate. Moreover, some authors also inspected the regularity of solutions to linear degenerate elliptic equations with drift term by establishing singular integral estimates. For example, Folland and Stein in [3] established $L^{p}$ estimates and Lipschitz estimates to the operator on the Heisenberg group

$$
L=-\frac{1}{4} \sum_{j=1}^{n}\left(X_{j}^{2}+Y_{j}^{2}\right)+i \alpha T
$$

for suitable $\alpha$, where $T$ is the vertical vector field. To the nondivergence linear degenerate elliptic operator

$$
£=\sum_{i, j=1}^{q} a_{i j}(x) X_{i} X_{j}+a_{0}(x) X_{0}
$$

constructed by Hörmander's vector fields, Bramanti and Zhu in [4] established $L^{p}$ estimates with $a_{i j}(x)$ and $a_{0}(x)$ belonging to $\mathrm{VMO}$ spaces related to $\left\{X_{1}, X_{2}, \cdots, X_{q}, X_{0}\right\}$ and Schauder estimates with $a_{i j}(x)$ and $a_{0}(x)$ being in Hölder spaces for strong solutions. It is important in [4] that the difference between equations without $X_{0}$ and with $X_{0}$ was pointed out. When $X_{1}, X_{2}, \cdots, X_{q}$ in (3) is basis vector fields and $X_{0}$ is the drift vector field on homogeneous groups, many scholars have obtained regularities to the operator $£$ with coefficients $a_{i j}$ and $a_{0}$ satisfying appropriate conditions, such as [5-8]. In addition, Austin and Tyson in [9] achieved the $C^{\infty}$-smoothness for the operator on the Heisenberg group $\mathbb{H}^{n}$

$$
L=-\frac{1}{4} \sum_{i=1}^{n}\left(X_{j}^{2}+Y_{\mathrm{j}}^{2}\right) \pm \sqrt{3} T
$$

by using the geometric analysis method.

Note that the equations studied in the above-cited papers are linear. In this paper, we consider the regularity to the 
weak solution of discontinuous subelliptic systems with drift term $T u$ on $\mathbb{H}^{n}$

$-\sum_{i=1}^{2 n} X_{i} A_{i}^{k}\left(x, u, \nabla_{H} u\right)-T u=B^{k}\left(x, u, \nabla_{H} u\right), \quad x \in \Omega, k=1,2, \cdots, N$

where $\Omega$ is the bounded domain in $\mathbb{H}^{n}$, $A_{i}^{k}$ belongs to the vanishing mean oscillation space (which is abbreviated as VMO) and satisfies the ellipticity on $\mathbb{R}^{2 n \times N}$ and polynomial growth conditions with the growth index $1<p<2$ for $\nabla_{H} u$, and also $A_{i}^{k}$ is continuous for $u$ and differentiable for $\nabla_{H} u$ with continuous derivatives,

$$
\nabla_{H} u=\left(X_{1} u, X_{2} u, \cdots X_{2 n} u\right)
$$

$X_{i}(i=1,2, \cdots, 2 n)$ is the horizontal vector field and $T$ is the vertical vector field in $\mathbb{H}^{n}$. For more information about $\mathbb{H}^{n}$, see Section 2. The nonhomogeneous term $B^{k}$ satisfies the controllable growth condition or natural growth condition. We will use the $A$-harmonic approximation method to conclude the partial Hölder regularity to the weak solutions and the partial Morrey regularity to the horizontal gradients of the weak solutions.

More regularity for the elliptic system without drift term, one can refer to [10-12] (Euclidean space) and [13-15] (Heisenberg group).

Now, for any $x \in \Omega, u, u_{0} \in \mathbb{R}^{N}, P, P_{0} \in \mathbb{R}^{2 n \times N}$, and the growth index $1<p<2$, we list the hypotheses that the system satisfies.

(H1). Let $A_{i}^{k}$ satisfy the following ellipticity and polynomial growth conditions (growth index $1<p<2$ ):

$$
\begin{gathered}
\left\langle D_{P} A_{i}^{k}(\mathrm{x}, u, P) P_{0}, P_{0}\right\rangle \geq \lambda(1+|P|)^{p-2}\left|P_{0}\right|^{2}, \\
\left|A_{i}^{k}(x, u, P)\right|+(1+|P|)\left|D_{P} A_{i}^{k}(x, u, P)\right| \leq \Lambda(1+|P|)^{p-1},
\end{gathered}
$$

where $D_{P} A_{i}^{k}$ denote the usual derivative of $A_{i}^{k}$ with respect to the variable $P, 0<\lambda \leq 1 \leq \Lambda<\infty$.

(H2). Assume that $A_{i}^{k}(x, u, P) /(1+|P|)^{p-1}$ is continuous for $u$. More precisely, there exists a bounded, concave, and nondecreasing continuous modulus $\omega:[0, \infty) \longrightarrow[0,1]$ with $\lim _{s \longrightarrow 0} \omega(s)=0=\omega(0)$ such that

$$
\left|A_{i}^{k}(x, u, P)-A_{i}^{k}\left(x, u_{0}, P\right)\right| \leq \Lambda \omega\left(\left|u-u_{0}\right|^{p}\right)(1+|P|)^{p-1} .
$$

(H3). Let $A_{i}^{k}$ be differentiable for the variable $P$ with continuous derivatives, that is, there exists a bounded, concave, and nondecreasing continuous modulus $\vartheta:[0, \infty) \longrightarrow[0,1]$ with $\vartheta(s) \leq s, \lim _{s \rightarrow 0} \vartheta(s)=0=\vartheta(0)$ such that

$$
\begin{aligned}
& \left|D_{P} A_{i}^{k}(x, u, P)-D_{P} A_{i}^{k}\left(x, u, P_{0}\right)\right| \\
& \quad \leq \Lambda \vartheta\left(\frac{\left|P-P_{0}\right|}{1+|P|+\left|P_{0}\right|}\right)\left(1+|P|+\left|P_{0}\right|\right)^{p-2} .
\end{aligned}
$$

(H4). For all $x \in B_{r}\left(x_{0}\right), A_{i}^{k}(x, u, P) /(1+|P|)^{p-1}$ satisfies the following VMO condition:

$$
\left|A_{i}^{k}(x, u, P)-\left(A_{i}^{k}(\cdot, u, P)\right)_{x_{0}, r}\right| \leq v_{x_{0}}(x, r)(1+|P|)^{p-1},
$$

where $v_{x_{0}}: \mathbb{R}^{2 n+1} \times\left[0, r_{0}\right] \longrightarrow[0,2 \Lambda]$ is a bounded function and satisfies

$$
\lim _{r_{0} \longrightarrow 0} v\left(r_{0}\right)=\lim _{r_{0} \longrightarrow 0} \sup _{x_{0} \in \Omega 0 \leq r \leq r_{0}} \sup _{0}\left(v_{x_{0}}(x, r)\right)_{B_{r}\left(x_{0}\right) \cap \Omega}=0 .
$$

Here, we have used in (11) and (12) the notation

$$
(f)_{x_{0}, r}=\frac{1}{\left|B_{r}\left(x_{0}\right)\right|} \int_{B_{r}\left(x_{0}\right)} f d x
$$

(HC) (controllable growth condition). The nonhomogeneous term $B^{k}$ satisfies the following controllable growth condition

$$
\left|B^{k}(x, u, P)\right| \leq c\left(1+|u|^{p^{*}-1}+|P|^{p\left(1-\left(1 / p^{*}\right)\right)}\right),
$$

where $c$ is a positive constant,

$$
p^{*}=\frac{p \wp}{\wp-p} \text { for } 1<p<\wp
$$

and $\wp$ denotes the homogeneous dimension of the Heisenberg group.

Obviously, we can see that system (5) includes the system

$$
\begin{aligned}
& -\sum_{i=1}^{2 n} X_{i}\left(A_{i}^{k}(x)\left(1+\left|\nabla_{H} u\right|^{2}\right)^{(p-2) / 2} X_{i} u^{k}\right) \\
& \quad-T u=B^{k}\left(x, u, \nabla_{H} u\right), \quad x \in \Omega, k=1,2, \cdots, N .
\end{aligned}
$$

We state the main result.

Theorem 1. Assume that $A_{i}^{k}\left(x, u, \nabla_{H} u\right)$ and $B^{k}\left(x, u, \nabla_{H} u\right)$ satisfy the assumptions (H1)-(H4) and (HC). If $1<p<2$ and $u \in H W^{1,2}\left(\Omega, \mathbb{R}^{N}\right)$ is a weak solution to system (5), i.e., for all $\varphi \in H W_{0}^{1,2}(\Omega)$,

$$
\int_{\Omega} A_{i}^{k}\left(x, u, \nabla_{H} u\right) \nabla_{H} \varphi d x+\int_{\Omega} u \cdot T \varphi d x=\int_{\Omega} B^{k}\left(x, u, \nabla_{H} u\right) \varphi d x,
$$

then, there exists a relatively closed singular set $\Omega_{0} \subset \Omega$ such that for any $\alpha \in(0,1)$, we have 


$$
u \in C_{l o c}^{0, \alpha}\left(\Omega \backslash \Omega_{0}, \mathbb{R}^{N}\right) .
$$

Moreover, for any $\beta=\wp-p(1-\alpha)$, we have

$$
\nabla_{H} u \in L_{l o c}^{p, \beta}\left(\Omega \backslash \Omega_{0}, \mathbb{R}^{2 n \times N}\right),
$$

where $L_{l o c}^{p, \beta}$ is a local Morrey space. The singular set $\Omega_{0}$ satisfies

$$
\left|\Omega_{0}\right|=0, \Omega_{0} \subset \sum_{1} \bigcup \sum_{2}
$$

where

$$
\begin{aligned}
& \sum_{1}=\left\{x_{0} \in \Omega: \limsup _{r \rightarrow 0}\left|\left(\nabla_{H} u\right)_{x_{0}, r}\right|=\infty\right\}, \\
& \sum_{2}=\left\{x_{0} \in \Omega: \liminf _{r \longrightarrow 0} \frac{1}{\left|B_{r}\left(x_{0}\right)\right|} \int_{B_{r}\left(x_{0}\right)}\left|\nabla_{H} u-\left(\nabla_{H} u\right)_{x_{0}, r}\right|^{2} d x>0\right\} .
\end{aligned}
$$

Corollary 2. Assume that $A_{i}^{k}\left(x, u, \nabla_{H} u\right)$ and $B^{k}\left(x, u, \nabla_{H} u\right)$ satisfy the assumptions (H1)-(H4) and the following assumption:

(HN). The nonhomogeneous term $B^{k}\left(x, u, \nabla_{H} u\right)$ satisfies the following $p$ natural growth condition

$$
\left|B^{k}(x, u, P)\right| \leq a|P|^{p}+b
$$

for $|u| \leq M$, where $a$ and $b$ are constants depending only on $M$.

Then, we have

$$
u \in C_{l o c}^{0, \alpha}\left(\Omega \backslash \Omega_{0}, \mathbb{R}^{N}\right) \text { and } \nabla_{H} u \in L_{l o c}^{p, \beta}\left(\Omega \backslash \Omega_{0}, \mathbb{R}^{2 n \times N}\right)
$$

for weak solution $u \in H W^{1,2}\left(\Omega, \mathbb{R}^{N}\right)$ to system (5) under the assumption $2 a M<\lambda$, where $1<p<2$ and $\Omega_{0}$ is same as in Theorem 1.

Its proof is direct by combining the proof of Theorem 1 in this paper with the proof of Theorem 1.2 in [15].

Let us recall that the $A$-harmonic approximation method was first introduced by Duzaar and Steffen in [16] and then extended to other cases by some authors, see [17-19]. In this paper, we use the $A$-harmonic approximation method described in [15] to conclude Theorem 1. Different from [15], the system considered by us has a drift term, which brings new challenges to our research. Actually, the processing of drift term are different from that the processings of other terms in the system. Moreover, Lemmas 11-14 in Section 3 used in proving Theorem 1 are different from the corresponding lemmas in [15] and will be rebuilt.

This paper is organized as follows: in Section 2, we introduce the related knowledge of the Heisenberg group, some function spaces on the Heisenberg group, horizontal affine functions, and some necessary lemmas. In Section 3, we show a Caccioppoli-type inequality for weak solution to
(5), the approximately $A$-harmonic lemma, the decay estimate, and iteration relations. In Section 4, the proof of Theorem 1 is given.

\section{Preliminaries}

2.1. The Heisenberg Group $\mathbb{H}^{n}$ and Some Function Spaces on $\mathbb{H}^{n}$. The Euclidean space $\mathbb{R}^{2 n+1}, n \geq 1$ with the group multiplication

$$
x \circ y=\left(x_{1}+y_{1}, x_{2}+y_{2}, \cdots, x_{2 n}+y_{2 n}, t+s+\frac{1}{2} \sum_{i=1}^{n}\left(x_{i} y_{n+i}-x_{n+i} y_{i}\right)\right) \text {, }
$$

where $x=\left(x_{1}, x_{2}, \cdots, x_{2 n}, t\right), y=\left(y_{1}, y_{2}, \cdots, y_{2 n}, s\right) \in \mathbb{R}^{2 n+1}$ leads to the Heisenberg group $\mathbb{H}^{n}$. The left invariant vector fields generated by commutation the Lie algebra on $\mathrm{H}^{n}$ are

$$
X_{i}=\partial_{x_{i}}-\frac{x_{n+i}}{2} \partial_{t}, X_{n+i}=\partial_{x_{n+i}}+\frac{x_{i}}{2} \partial_{t}, 1 \leq i \leq n,
$$

and the only nontrivial commutator of such fields is

$$
T=\partial_{t}=\left[X_{i}, X_{n+i}\right]=X_{i} X_{n+i}-X_{n+i} X_{i}, 1 \leq i \leq n
$$

We call that $X_{1}, X_{2}, \cdots, X_{2 n}$ are the horizontal vector fields on $\mathbb{H}^{n}$ and $T$ the vertical vector field. Denote the horizontal gradient of a smooth function $u$ on $\mathbb{H}^{n}$ by

$$
\nabla_{H} u=\left(X_{1} u, X_{2} u, \cdots, X_{2 n} u\right)
$$

The homogeneous dimension of $\mathbb{H}^{n}$ is $\wp=2 n+2$. The Haar measure in $\mathbb{H}^{n}$ is equivalent to the Lebesgue measure in $\mathbb{R}^{2 n+1}$. We denote the Lebesgue measure of a measurable set $E \subset \mathbb{H}^{n}$ by $|E|$.

The Carnot-Carathèodary metric ( $\mathrm{C}-\mathrm{C}$ metric) between two points in $\mathbb{H}^{n}$ is the shortest length of the horizontal curve joining them, denoted by $d$. The ball induced by the $\mathrm{C}-\mathrm{C}$ metric is

$$
B_{\rho}(x)=\left\{y \in \mathbb{H}^{n}: d(y, x)<\rho\right\}
$$

For $x=\left(x_{1}, x_{2}, \cdots, x_{2 n}, t\right) \in \mathbb{H}^{n}$, its Korànyi metric is denoted by

$$
\|x\|_{\mathbb{H}^{n}}=\left(\left(\sum_{i=1}^{2 n} x_{i}^{2}\right)^{2}+t^{2}\right)^{1 / 4}
$$

The $\mathrm{C}-\mathrm{C}$ metric $d$ is equivalent to the Korànyi metric

$$
d(x, y)=\left\|x^{-1} \circ y\right\|_{\mathbb{H}^{n}} .
$$

For $1 \leq p<\infty, \Omega \subset \mathbb{H}^{n}$, the horizontal Sobolev space $H W^{k, p}(\Omega)$ is defined as 
$H W^{k, p}(\Omega)=\left\{u \in L^{p}(\Omega): \nabla_{H} u \in L^{p}(\Omega), \nabla_{H}^{2} u \in L^{p}(\Omega), \cdots, \nabla_{H}^{k} u \in L^{p}(\Omega)\right\}$,

which is a Banach space under the norm

$$
\|u\|_{H W^{k, p}(\Omega)}=\|u\|_{L^{p}(\Omega)}+\sum_{m=1}^{k}\left\|\nabla_{H}^{m} u\right\|_{L^{p}(\Omega)} .
$$

The local horizontal Sobolev space $H W_{\text {loc }}^{k, p}(\Omega)$ is

$$
H W_{\mathrm{loc}}^{k, p}(\Omega):=\left\{u: u \in H W^{k, p}\left(\Omega^{\prime}\right), \forall \Omega^{\prime} \subset \subset \Omega\right\},
$$

and the space $H W_{0}^{k, p}(\Omega)$ is the closure of $C_{0}^{\infty}(\Omega)$ in $H$ $W^{k, p}(\Omega)$.

Similar to the definition in [20], Morrey space and Campanato space on Heisenberg group are defined as follows.

Definition 3 (Morrey space). Let $1 \leq p<\infty$ and $\beta \geq 0$. For the function $g \in L^{p}(\Omega)$, if

$$
\|g\|_{L^{p, \beta}(\Omega)}:=\left(\sup _{x \in \Omega, 0<r<\operatorname{diam} \Omega} r^{-\beta} \int_{\Omega(x, r)}|g(y)|^{p} d y\right)^{1 / p}<\infty,
$$

then, we say that $g$ belongs to the Morrey space denoted by $L^{p, \beta}(\Omega)$, where $\Omega(x, r)=\Omega \cap B_{r}(x)$.

Definition 4 (Campanato space). Let $1 \leq p<\infty$ and $\beta \geq 0$. For the function $g \in L^{p}(\Omega)$, if

$$
[g]_{p, \beta}:=\left(\sup _{x \in \Omega, 0<r<\operatorname{diam} \Omega} r^{-\beta} \int_{\Omega(x, r)}\left|g(y)-(g)_{\Omega(x, r)}\right|^{p} d y\right)^{1 / p}<\infty,
$$

then, we say that $g$ belongs to the Campanato space denoted by $L^{p, \beta}(\Omega)$, and its norm is defined as

$$
\|g\|_{L^{p, \beta}(\Omega)}=[g]_{p, \beta}+\|g\|_{L^{p}}
$$

Lemma 5 (see [21, 22]). If for any $1<p<\infty, 0<\alpha<1$, we have $g \in L^{p, \wp+p \alpha}(\Omega)$, then $g \in C^{0, \alpha}(\Omega)$.

Lemma 6 (Sobolev inequality, [23]). For $B_{r} \subset \mathbb{H}^{n}, 1 \leq q<$ $\wp=2 n+2$ and for any $u \in H W_{0}^{1, q}\left(B_{r}\right)$, it holds

$$
\left(\frac{1}{\left|B_{r}\right|} \int_{B_{r}}|u|^{(\wp q) /(\wp-q)} d x\right)^{(\wp-q) /(\wp q)} \leq c r\left(\frac{1}{\left|B_{r}\right|} \int_{B_{r}}\left|\nabla_{H} u\right|^{q} d x\right)^{1 / q}
$$

where $c=c(\wp, q)>0$.

Then, the following four lemmas are true.
For the proof of Lemma 6, see [24] and [25].

2.2. Horizontal Affine Function and Some Lemmas. Let $u \in$ $L^{2}\left(B_{r}\left(x_{0}\right), \mathbb{R}^{N}\right), x$ and $x_{0} \in \mathbb{R}^{2 n+1}$. Denote the horizontal components of $x, x_{0}$ by

$$
\begin{aligned}
x^{\prime} & =\left(x_{1}, \cdots, x_{2 n}\right), \\
x_{0}^{\prime} & =\left(x_{1}^{0}, \cdots, x_{2 n}^{0}\right) .
\end{aligned}
$$

Let $l: \mathbb{R}^{2 n} \longrightarrow \mathbb{R}^{N}$ be a horizontal affine function. Following [13], if the horizontal affine function

$$
l_{x_{0}, r}\left(x^{\prime}\right)=l_{x_{0}, r}\left(x_{0}^{\prime}\right)+\nabla_{H} l_{x_{0}, r}\left(x^{\prime}-x_{0}^{\prime}\right)
$$

is a minimizer of the functional

$$
l \longrightarrow \frac{1}{\left|B_{r}\left(x_{0}\right)\right|} \int_{B_{r}\left(x_{0}\right)}|u-l|^{2} d x
$$

then, we have

$$
\begin{aligned}
l_{x_{0}, r}\left(x_{0}^{\prime}\right) & =u_{x_{0}, r}=\frac{1}{\left|B_{r}\left(x_{0}\right)\right|} \int_{B_{r}\left(x_{0}\right)} u d x, \\
\nabla_{H} l_{x_{0}, r} & =\frac{\wp-2}{c_{0} \wp} \frac{\wp+2}{r^{2}} \frac{1}{\left|B_{r}\left(x_{0}\right)\right|} \int_{B_{r}\left(x_{0}\right)} u \otimes\left(x^{\prime}-x_{0}^{\prime}\right) d x,
\end{aligned}
$$

where $u \otimes\left(x^{\prime}-x_{0}^{\prime}\right)$ stands for the matrix $\left[u^{k}\left(x_{i}-x_{i}^{0}\right)\right]_{N \times 2 n}$, $k=1, \cdots, N, i=1, \cdots, 2 n$, and $c_{0}$ is a positive constant defined as

$c_{0}=\frac{\int_{0}^{\pi}(\sin \theta)^{n} d \theta}{\int_{0}^{\pi}(\sin \theta)^{n-1} d \theta}= \begin{cases}\frac{[(2 m-2) ! !]^{2}}{(2 m-1) ! !(2 m-3) ! !} \frac{2}{\pi}, & n=2 m-1, \\ \frac{[(2 m-1) ! !]^{2}}{(2 m) ! !(2 m-2) ! !} \frac{\pi}{2}, & n=2 m .\end{cases}$

According to the meaning of $l_{x_{0}, r}$, one has the following Poincaré inequality ([13]):

$$
\begin{aligned}
& \left(\frac{1}{\left|B_{r}\left(x_{0}\right)\right|} \int_{B_{r}\left(x_{0}\right)}\left|u-l_{x_{0}, r}\left(x^{\prime}\right)\right|^{p} d x\right)^{1 / p} \\
& \leq C_{p} r\left(\frac{1}{\left|B_{r}\left(x_{0}\right)\right|} \int_{B_{r}\left(x_{0}\right)}\left|\nabla_{H} u-\nabla_{H} l_{x_{0}, r}\right|^{q} d x\right)^{1 / q},
\end{aligned}
$$

where $1<q<\wp, 1 \leq p \leq(q \wp) /(\wp-q)$.

Throughout the paper, we define

$$
V(\varsigma)=\left(1+|\varsigma|^{2}\right)^{(p-2) / 4} \varsigma, 1<p<2, \zeta \in \mathbb{R}^{N}
$$

Lemma 7 (see [26]). For any $\zeta_{1}, \zeta_{2} \in \mathbb{R}^{N}$ and $s>0$, it holds 
(1) $2^{-1 / 2} \min \left(\left|\zeta_{1}\right|,\left|\zeta_{1}\right|^{p / 2}\right) \leq 2^{(p-2) / 4} \min \left(\left|\zeta_{1}\right|,\left|\zeta_{1}\right|^{p / 2}\right) \leq 1$ $V\left(\zeta_{1}\right) \mid \leq \min \left(\left|\zeta_{1}\right|,\left|\zeta_{1}\right|^{p / 2}\right)$

(2) $\left|V\left(s \zeta_{1}\right)\right| \leq \max \left(s, s^{p / 2}\right)\left|V\left(\zeta_{1}\right)\right|$

(3) $\left|V\left(\zeta_{1}+\zeta_{2}\right)\right| \leq c_{p}\left(\left|V\left(\zeta_{1}\right)\right|+\left|V\left(\zeta_{2}\right)\right|\right)$

(4) $(p / 2)\left|\zeta_{1}-\zeta_{2}\right| \leq\left|V\left(\zeta_{1}\right)-V\left(\zeta_{2}\right)\right| /$ $\left(1+\left|\zeta_{1}\right|^{2}+\left|\zeta_{2}\right|^{2}\right)^{(p-2) / 4} \leq c(p, k)\left|\zeta_{1}-\zeta_{2}\right|$

(5) $\left|V\left(\zeta_{1}\right)-V\left(\zeta_{2}\right)\right| \leq c(p, k)\left|V\left(\zeta_{1}-\zeta_{2}\right)\right|$

(6) $\left|V\left(\zeta_{1}-\zeta_{2}\right)\right| \leq c(p, M)\left|V\left(\zeta_{1}\right)-V\left(\zeta_{2}\right)\right|$ for $\zeta_{2}$ with $\mid \zeta_{2}$ $\mid \leq M$

Lemma 8 (Sobolev-Poincaré-type inequality, [15]). Let $1<$ $p<2$ and $u \in H W^{1, p}\left(B_{r}\left(x_{0}\right), \mathbb{R}^{N}\right)$ with $B_{r}\left(x_{0}\right) \subset \Omega$. Then, it follows

$$
\begin{aligned}
& \left(\frac{1}{\left|B_{r}\left(x_{0}\right)\right|} \int_{B_{r}\left(x_{0}\right)}\left|V\left(\frac{u-u_{x_{0}, r}}{r}\right)\right|^{2 p^{*} / p} d x\right)^{p / 2 p^{*}} \\
& \leq C_{p}\left(\frac{1}{\left|B_{r}\left(x_{0}\right)\right|} \int_{B_{r}\left(x_{0}\right)}\left|V\left(\nabla_{H} u\right)\right|^{2} d x\right)^{1 / 2},
\end{aligned}
$$

where $p^{*}=(p \wp) /(\wp-p)$ and $C_{p}$ depends only on $\wp, N$, p. In particular, we have

$$
\begin{aligned}
& \left(\frac{1}{\left|B_{r}\left(x_{0}\right)\right|} \int_{B_{r}\left(x_{0}\right)}\left|V\left(\frac{u-u_{x_{0}, r}}{r}\right)\right|^{2} d x\right)^{1 / 2} \\
& \leq C_{p}\left(\frac{1}{\left|B_{r}\left(x_{0}\right)\right|} \int_{B_{r}\left(x_{0}\right)}\left|V\left(\nabla_{H} u\right)\right|^{2} d x\right)^{1 / 2} .
\end{aligned}
$$

Let $A \in \operatorname{Bil}\left(\Omega \times \mathbb{R}^{N} \times \mathbb{R}^{2 n \times N}, \mathbb{R}^{2 n \times N}\right)$ be a bilinear form with constant tensorial coefficients. We recall that a map $h$ $\in C^{\infty}\left(B_{r}\left(x_{0}\right), \mathbb{R}^{N}\right)$ is A-harmonic if and only if it holds

$$
\frac{1}{\left|B_{r}\left(x_{0}\right)\right|} \int_{B_{r}\left(x_{0}\right)} A\left(\nabla_{H} h, \nabla_{H} \phi\right) d x=0
$$

for any testing function $\phi \in C_{0}^{\infty}\left(B_{r}\left(x_{0}\right), \mathbb{R}^{N}\right)$.

Lemma 9 (see [15]). Let $h \in H W^{1,1}\left(\Omega, \mathbb{R}^{N}\right)$ be a weak solution of the constant coefficient system

$$
-\sum_{i=1}^{2 n} X_{i} A_{i}^{k}\left(\nabla_{H} h\right)=0, \quad k=1, \cdots, N
$$

Then, $h$ is smooth and there exists $c \geq 1$ such that for any $B_{r}\left(x_{0}\right) \subset \Omega$,

$$
\sup _{B_{r / 2}\left(x_{0}\right)}\left(\left|\nabla_{H} h\right|^{2}+\left|\nabla_{H}^{2} h\right|^{2}\right) \leq c r^{-2} \frac{1}{\left|B_{r}\left(x_{0}\right)\right|} \int_{B_{r}\left(x_{0}\right)}\left|\nabla_{H} h\right|^{2} d x .
$$

Lemma 10 (see [15]). Given $0<v \leq L, 1<p<2$, for any $\varepsilon$ $>0$, there exist constants $\rho \in[0,1]$ and $\delta=\delta(\wp, N, p, v, L, \varepsilon)$ $\in(0,1]$ and a bilinear form $A$ on $\mathbb{R}^{2 n \times N}$ satisfying that for $P, \bar{P} \in \mathbb{R}^{2 n \times N}$,

$$
\begin{aligned}
& A(P, P) \geq v|P|^{2}, \\
& A(P, \bar{P}) \leq L|P||\bar{P}| .
\end{aligned}
$$

If $w \in H W^{1, p}\left(B_{r}\left(x_{0}\right), \mathbb{R}^{N}\right)$ is an approximate A-harmonic map, i.e., for any $\phi \in C_{0}^{\infty}\left(B_{r}\left(x_{0}\right), \mathbb{R}^{N}\right)$, it holds

$$
\begin{aligned}
& \frac{1}{\left|B_{r}\left(x_{0}\right)\right|} \int_{B_{r}\left(x_{0}\right)} A\left(\nabla_{H} w, \nabla_{H} \phi\right) d x \leq \delta \rho \sup _{B_{r}\left(x_{0}\right)}\left|\nabla_{H} \phi\right|, \\
& \frac{1}{\left|B_{r}\left(x_{0}\right)\right|} \int_{B_{r}\left(x_{0}\right)}\left|V\left(\nabla_{H} w\right)\right| d x \leq \rho^{2},
\end{aligned}
$$

then, there exists a A-harmonic map $h \in C^{\infty}\left(B_{r}\left(x_{0}\right)\right.$, $\left.\mathbb{R}^{N}\right)$ satisfying

$$
\begin{aligned}
& \frac{1}{\left|B_{r}\left(x_{0}\right)\right|} \int_{B_{r}\left(x_{0}\right)}\left|V\left(\frac{w-\rho h}{r}\right)\right|^{2} d x \leq \rho^{2} \varepsilon, \\
& \frac{1}{\left|B_{r}\left(x_{0}\right)\right|} \int_{B_{r}\left(x_{0}\right)}\left|V\left(\nabla_{H} h\right)\right|^{2} d x \leq 1 .
\end{aligned}
$$

\section{Some Lemmas}

For convenience, we introduce some notations:

$$
p^{\prime}=\frac{p}{p-1},
$$

$$
p^{*}=\frac{p \wp}{\wp-p}
$$

$$
\left(p^{*}\right)^{\prime}=\frac{p^{*}}{p^{*}-1},
$$

$$
\begin{aligned}
f\left(x_{0}, r\right)= & r^{p^{\prime}}\left(\frac{1}{\left|B_{r}\left(x_{0}\right)\right|} \int_{B_{r}\left(x_{0}\right)}\left(\left|\nabla_{H} u\right|^{p}+|u|^{p^{*}}+1\right) d x\right)^{p^{\prime} /\left(p^{*}\right)^{\prime}} \\
& +r^{2}\left(\frac{1}{\left|B_{r}\left(x_{0}\right)\right|} \int_{B_{r}\left(x_{0}\right)}\left(\left|\nabla_{H} u\right|^{p}+|u|^{p^{*}}+1\right) d x\right)^{2 /\left(p^{*}\right)^{\prime}}, \\
\Phi(r):= & \Phi\left(x_{0}, r, l\right):=\frac{1}{\left|B_{r}\left(x_{0}\right)\right|} \int_{B_{r}\left(x_{0}\right)}\left|V\left(\nabla_{H} u-\nabla_{H} l\right)\right|^{2} d x, \\
\Psi(r):= & \Psi\left(x_{0}, r, l\right):=\frac{1}{\left|B_{r}\left(\mathrm{x}_{0}\right)\right|} \int_{B_{r}\left(x_{0}\right)}\left|V\left(\frac{u-l}{r}\right)\right|^{2} d x \\
& +\frac{1}{\left|B_{r}\left(x_{0}\right)\right|} \int_{B_{r}\left(x_{0}\right)}\left|\frac{u-l}{r}\right|^{2} d x,
\end{aligned}
$$




$$
\begin{aligned}
\Psi_{*}(r):= & \Psi_{*}\left(x_{0}, r, l\right):=\Psi\left(x_{0}, r, l\right) \\
& +\omega\left(\frac{1}{\left|B_{r}\left(x_{0}\right)\right|} \int_{B_{r}\left(x_{0}\right)}\left|u-l\left(x_{0}^{\prime}\right)\right|^{p} d x\right) \\
& +v(r)+f\left(x_{0}, r\right),
\end{aligned}
$$

where

$$
v(r)=\sup _{x_{0} \in \Omega_{0 \leq r^{\prime} \leq r}} \sup _{0}\left(v_{x_{0}}\left(x, r^{\prime}\right)\right)_{B_{r}{ }^{\prime}\left(x_{0}\right) \cap \Omega} .
$$

Lemma 11 (Caccioppoli-type inequality). Let $u \in H W^{1,2}(\Omega$ , $\mathbb{R}^{N}$ ) be a weak solution to (5) under the assumptions (H1)-(H4) and (HC). Then, for any $x_{0}=\left(x_{0}^{\prime}, t_{0}\right) \in \Omega$, $B_{r}\left(x_{0}\right) \subset \subset \Omega$ and the horizontal affine function $l: \mathbb{R}^{2 n} \longrightarrow$ $\mathbb{R}^{N}$ with $\left|l\left(x_{0}^{\prime}\right)\right|+\left|\nabla_{H} l\right| \leq M_{0}$, we have

$$
\Phi\left(x_{0}, \frac{r}{2}, l\right) \leq C_{c} \Psi_{*}\left(x_{0}, r, l\right)
$$
$M_{0}$.

where $C_{c}$ is a positive constant depending on $\wp, p, \lambda, \Lambda$,

Proof. We choose a standard cut-off function $\eta \in C_{0}^{\infty}\left(B_{r}\left(x_{0}\right)\right.$, $[0,1])$ with $\eta \equiv 1$ on $B_{r / 2}\left(x_{0}\right)$ and

$$
\begin{aligned}
\left|\nabla_{H} \eta\right| & \leq \frac{4}{r}, \\
|T \eta| & \leq \frac{c}{r^{2}} .
\end{aligned}
$$

Taking a testing function $\varphi=\eta^{2}(u-l)$ in (17), we have

$$
\begin{aligned}
& \int_{B_{r}\left(x_{0}\right)} \eta^{2} A_{i}^{k}\left(x, u, \nabla_{H} u\right)\left(\nabla_{H} u-\nabla_{H} l\right) d x \\
& =-2 \int_{B_{r}\left(x_{0}\right)} \eta A_{i}^{k}\left(x, u, \nabla_{H} u\right)(u-l) \nabla_{H} \eta d x \\
& \quad+\int_{B_{r}\left(x_{0}\right)} \eta^{2} B^{k}\left(x, u, \nabla_{H} u\right)(u-l) d x \\
& \quad-\int_{B_{r}\left(x_{0}\right)} u \cdot T\left(\eta^{2}(u-l)\right) d x
\end{aligned}
$$
yields

Dividing the equality above by the measure of the ball, it

$$
\begin{aligned}
& \frac{1}{\left|B_{r}\left(x_{0}\right)\right|} \int_{B_{r}\left(x_{0}\right)} \eta^{2} A_{i}^{k}\left(x, u, \nabla_{H} u\right)\left(\nabla_{H} u-\nabla_{H} l\right) d x \\
& =-2 \frac{1}{\left|B_{r}\left(x_{0}\right)\right|} \int_{B_{r}\left(x_{0}\right)} \eta A_{i}^{k}\left(x, u, \nabla_{H} u\right)(u-l) \nabla_{H} \eta d x \\
& \quad+\frac{1}{\left|B_{r}\left(x_{0}\right)\right|} \int_{B_{r}\left(x_{0}\right)} \eta^{2} B^{k}\left(x, u, \nabla_{H} u\right)(u-l) d x \\
& \quad-\frac{1}{\left|B_{r}\left(x_{0}\right)\right|} \int_{B_{r}\left(x_{0}\right)} u \cdot T\left(\eta^{2}(u-l)\right) d x .
\end{aligned}
$$

Note that $\left(A_{i}^{k}\left(\cdot, l\left(x_{0}^{\prime}\right), \nabla_{H} l\right)\right)_{x_{0}, r}$ is a constant, so it infers by using the integration by parts that

$$
0=\frac{1}{\left|B_{r}\left(x_{0}\right)\right|} \int_{B_{r}\left(x_{0}\right)}\left(A_{i}^{k}\left(\cdot, l\left(x_{0}^{\prime}\right), \nabla_{H} l\right)\right)_{x_{0}, r} \nabla_{H} \varphi d x
$$

Owing to

$$
\begin{gathered}
-\frac{1}{\left|B_{r}\left(x_{0}\right)\right|} \int_{B_{r}\left(x_{0}\right)} \eta^{2} A_{i}^{k}\left(x, u, \nabla_{H} l\right)\left(\nabla_{H} u-\nabla_{H} l\right) d x \\
=2 \frac{1}{\left|B_{r}\left(x_{0}\right)\right|} \int_{B_{r}\left(x_{0}\right)} \eta A_{i}^{k}\left(x, u, \nabla_{H} l\right)(u-l) \nabla_{H} \eta d x \\
\quad-\frac{1}{\left|B_{r}\left(x_{0}\right)\right|} \int_{B_{r}\left(x_{0}\right)} A_{i}^{k}\left(x, u, \nabla_{H} l\right) \nabla_{H} \varphi d x,
\end{gathered}
$$

we substitute the left and right hand sides of (60) and (61) into the left and right hand sides of (59), respectively, to obtain

$$
\begin{aligned}
& \frac{1}{\left|B_{r}\left(x_{0}\right)\right|} \int_{B_{r}\left(x_{0}\right)} \eta^{2}\left(A_{i}^{k}\left(x, u, \nabla_{H} u\right)-A_{i}^{k}\left(x, u, \nabla_{H} l\right)\right)\left(\nabla_{H} u-\nabla_{H} l\right) d x \\
& =2 \frac{1}{\left|B_{r}\left(x_{0}\right)\right|} \int_{B_{r}\left(x_{0}\right)} \eta\left(A_{i}^{k}\left(x, u, \nabla_{H} l\right)-A_{i}^{k}\left(x, u, \nabla_{H} u\right)\right)(u-l) \nabla_{H} \eta d x \\
& \quad-\frac{1}{\left|B_{r}\left(x_{0}\right)\right|} \int_{B_{r}\left(x_{0}\right)} A_{i}^{k}\left(x, u, \nabla_{H} l\right) \nabla_{H} \varphi d x \\
& \quad+\frac{1}{\left|B_{r}\left(x_{0}\right)\right|} \int_{B_{r}\left(x_{0}\right)}\left(A_{i}^{k}\left(\cdot, l\left(x_{0}^{\prime}\right), \nabla_{H} l\right)\right)_{x_{0}, r} \nabla_{H} \varphi d x \\
& \quad+\frac{1}{\left|B_{r}\left(x_{0}\right)\right|} \int_{B_{r}\left(x_{0}\right)} \eta^{2} B^{k}\left(x, u, \nabla_{H} u\right)(u-l) d x \\
& \quad-\frac{1}{\left|B_{r}\left(x_{0}\right)\right|} \int_{B_{r}\left(x_{0}\right)} u \cdot T\left(\eta^{2}(u-l)\right) d x .
\end{aligned}
$$

Then, adding and subtracting the same term $\left(1 /\left|B_{r}\left(x_{0}\right)\right|\right)$ $\int_{B_{r}\left(x_{0}\right)} A_{i}^{k}\left(x, l\left(x_{0}^{\prime}\right), \nabla_{H} l\right) \nabla_{H} \varphi d x$ on the right hand side of the above equality, it gets

$$
\begin{aligned}
I_{0}:= & \frac{1}{\left|B_{r}\left(x_{0}\right)\right|} \int_{B_{r}\left(x_{0}\right)} \eta^{2}\left(A_{i}^{k}\left(x, u, \nabla_{H} u\right)-A_{i}^{k}\left(x, u, \nabla_{H} l\right)\right)\left(\nabla_{H} u-\nabla_{H} l\right) d x \\
= & 2 \frac{1}{\left|B_{r}\left(x_{0}\right)\right|} \int_{B_{r}\left(x_{0}\right)} \eta\left(A_{i}^{k}\left(x, u, \nabla_{H} l\right)-A_{i}^{k}\left(x, u, \nabla_{H} u\right)\right)(u-l) \nabla_{H} \eta d x \\
& +\frac{1}{\left|B_{r}\left(x_{0}\right)\right|} \int_{B_{r}\left(x_{0}\right)}\left(A_{i}^{k}\left(x, l\left(x_{0}^{\prime}\right), \nabla_{H} l\right)-A_{i}^{k}\left(x, u, \nabla_{H} l\right)\right) \nabla_{H} \varphi d x \\
& +\frac{1}{\left|B_{r}\left(x_{0}\right)\right|} \int_{B_{r}\left(x_{0}\right)}\left(\left(A_{i}^{k}\left(\cdot, l\left(x_{0}^{\prime}\right), \nabla_{H} l\right)\right)_{x_{0}, r}\right. \\
& \left.-A_{i}^{k}\left(x, l\left(x_{0}^{\prime}\right), \nabla_{H} l\right)\right) \nabla_{H} \varphi d x+\frac{1}{\left|B_{r}\left(x_{0}\right)\right|} \int_{B_{r}\left(x_{0}\right)} \eta^{2} B^{k}\left(x, u, \nabla_{H} u\right)(u-l) d x \\
& -\frac{1}{\left|B_{r}\left(x_{0}\right)\right|} \int_{B_{r}\left(x_{0}\right)} u \cdot T\left(\eta^{2}(u-l)\right) d x=: 2 I_{1}+I_{2}+I_{3}+I_{4}+I_{5} .
\end{aligned}
$$

The treatments to the terms $I_{0}, I_{1}, \cdots, I_{4}$ in (63) are similar to that of Lemma 4.1 in [15], and we simply write the processes of proofs. By (7), (44) and the known inequality $(1+|a|+|b-a|)^{2} \leq 3\left(1+|a|^{2}+|b-a|^{2}\right)$, it gains 
$I_{0} \geq \lambda\left[3\left(1+M_{0}^{2}\right)\right]^{(p-2) / 2} \frac{1}{\left|B_{r}\left(x_{0}\right)\right|} \int_{B_{r}\left(x_{0}\right)} \eta^{2}\left|V\left(\nabla_{H} u-\nabla_{H} l\right)\right|^{2} d x$.

By (8), $\left|\nabla_{H} \eta\right| \leq 4 / r$, Young's inequality and Lemma 7 , we have

$$
\begin{aligned}
I_{1} \leq & 2 \varepsilon \frac{1}{\left|B_{r}\left(x_{0}\right)\right|} \int_{B_{r}\left(x_{0}\right)} \eta^{2}\left|V\left(\nabla_{H} u-\nabla_{H} l\right)\right|^{2} d x \\
& +c\left(p, \Lambda, M_{0}\right) \varepsilon^{1 /(1-p)} \frac{1}{\left|B_{r}\left(x_{0}\right)\right|} \int_{B_{r}\left(x_{0}\right)}\left|V\left(\frac{u-l}{r}\right)\right|^{2} d x .
\end{aligned}
$$

It implies from (9), Young's inequality, Jensen's inequality and Lemma 7 that

$$
\begin{aligned}
I_{2} \leq & 2 \varepsilon \frac{1}{\left|B_{r}\left(x_{0}\right)\right|} \int_{B_{r}\left(x_{0}\right)} \eta^{2}\left|V\left(\nabla_{H} u-\nabla_{H} l\right)\right|^{2} d x \\
& +2 \varepsilon \frac{1}{\left|B_{r}\left(x_{0}\right)\right|} \int_{B_{r}\left(x_{0}\right)}\left|V\left(\frac{u-l}{r}\right)\right|^{2} d x \\
& +c\left(p, \Lambda, M_{0}\right) \varepsilon^{1 /(1-p)} \omega\left(\frac{1}{\left|B_{r}\left(x_{0}\right)\right|} \int_{B_{r}\left(x_{0}\right)}\left|u-l\left(x_{0}^{\prime}\right)\right|^{p} d x\right) .
\end{aligned}
$$

Using (11), Young's inequality and Lemma 7, it follows

$$
\begin{aligned}
I_{3} \leq & 2 \varepsilon \frac{1}{\left|B_{r}\left(x_{0}\right)\right|} \int_{B_{r}\left(x_{0}\right)} \eta^{2}\left|V\left(\nabla_{H} u-\nabla_{H} l\right)\right|^{2} d x \\
& +2 \varepsilon \frac{1}{\left|B_{r}\left(x_{0}\right)\right|} \int_{B_{r}\left(x_{0}\right)}\left|V\left(\frac{u-l}{r}\right)\right|^{2} d x \\
& +c\left(p, \Lambda, M_{0}\right) \varepsilon^{1 /(1-p)} v(r) .
\end{aligned}
$$

We have by using (14), Hölder's inequality, Lemma 6 , Young's inequality and Lemma 7 that

$$
\begin{aligned}
I_{4} \leq & 4 c_{p} \varepsilon \frac{1}{\left|B_{r}\left(x_{0}\right)\right|} \int_{B_{r}\left(x_{0}\right)} \eta^{2}\left|V\left(\nabla_{H} u-\nabla_{H} l\right)\right|^{2} d x \\
& +4 c_{p} \varepsilon \frac{1}{\left|B_{r}\left(x_{0}\right)\right|} \int_{B_{r}\left(x_{0}\right)}\left|V\left(\frac{u-l}{r}\right)\right|^{2} d x+c(p, \varepsilon) r^{p^{\prime}} \\
& \cdot\left(\frac{1}{\left|B_{r}\left(x_{0}\right)\right|} \int_{B_{r}\left(x_{0}\right)}\left(\left|\nabla_{H} u\right|^{p}+|u|^{p^{*}}+1\right) d x\right)^{p^{\prime}\left(p^{*}\right)^{\prime}} \\
& +c(p, \varepsilon) r^{2}\left(\frac{1}{\left|B_{r}\left(x_{0}\right)\right|} \int_{B_{r}\left(x_{0}\right)}\left(\left|\nabla_{H} u\right|^{p}+|u|^{p^{*}}+1\right) d x\right)^{2 /\left(p^{*}\right)^{\prime}} .
\end{aligned}
$$

The remaining task is to deal with $I_{5}$. Noting $l$ is independent of $t$ and so

$$
T l=0,
$$

we use $|T \eta| \leq c / r^{2}$ to obtain

$$
\begin{aligned}
I_{5} & =-\frac{1}{\left|B_{r}\left(x_{0}\right)\right|} \int_{B_{r}\left(x_{0}\right)} u \cdot T\left(\eta^{2}(u-l)\right) d x \\
& =-\frac{1}{\left|B_{r}\left(x_{0}\right)\right|} \int_{B_{r}\left(x_{0}\right)}(u-l) \cdot T\left(\eta^{2}(u-l)\right) d x \\
& =-\frac{1}{\left|B_{r}\left(x_{0}\right)\right|} \int_{B_{r}\left(x_{0}\right)} \eta(u-l)^{2} T \eta d x \\
& \leq \frac{c}{\left|B_{r}\left(x_{0}\right)\right|} \int_{B_{r}\left(x_{0}\right)}\left|\frac{u-l}{r}\right|^{2} d x .
\end{aligned}
$$

Now, substituting (64)-(70) into (63), and taking $\varepsilon$ small enough, it implies

$$
\begin{aligned}
& \frac{1}{\left|B_{r / 2}\left(x_{0}\right)\right|} \int_{B_{r / 2}\left(x_{0}\right)}\left|V\left(\nabla_{H} u-\nabla_{H} l\right)\right|^{2} d x \\
& \leq C_{c}\left[\frac{1}{\left|B_{r}\left(x_{0}\right)\right|} \int_{B_{r}\left(x_{0}\right)}\left|V\left(\frac{u-l}{r}\right)\right|^{2} d x+\frac{1}{\left|B_{r}\left(x_{0}\right)\right|} \int_{B_{r}\left(x_{0}\right)}\left|\frac{u-l}{r}\right|^{2} d x\right] \\
& \quad+C_{c} \omega\left(\frac{1}{\left|B_{r}\left(x_{0}\right)\right|} \int_{B_{r}\left(x_{0}\right)}\left|u-l\left(x_{0}^{\prime}\right)\right|^{p} d x\right)+C_{c} v(r) \\
& \quad+C_{c} r^{p^{\prime}}\left(\frac{1}{\left|B_{r}\left(x_{0}\right)\right|} \int_{B_{r}\left(x_{0}\right)}\left(\left|\nabla_{H} u\right|^{p}+|u|^{p^{*}}+1\right) d x\right)^{p^{\prime}\left(p^{*}\right)^{\prime}} \\
& \quad+C_{c} r^{2}\left(\frac{1}{\left|B_{r}\left(x_{0}\right)\right|} \int_{B_{r}\left(x_{0}\right)}\left(\left|\nabla_{H} u\right|^{p}+|u|^{p^{*}}+1\right) d x\right)^{2 /\left(p^{*}\right)^{\prime}} .
\end{aligned}
$$

Then, (56) is proved.

Lemma 12 (approximately $A$-harmonic lemma). Assume the assumptions of Theorem 1 are satisfied. For $B_{2 r}\left(x_{0}\right) \subset \Omega$ with $r \leq r_{0}$ and a horizontal affine function $l: \mathbb{R}^{2 n} \longrightarrow \mathbb{R}^{N}$ with $\mid$ $l\left(x^{\prime}{ }_{0}\right)|+| \nabla_{H} l \mid \leq M_{0}$, we define

$$
\begin{aligned}
& A=\left(D_{P} A_{i}^{k}\left(\cdot, l\left(x_{0}^{\prime}\right), \nabla_{H} l\right)\right)_{x_{0}, r}, \\
& w=u-l
\end{aligned}
$$

then, for all $\phi \in C_{0}^{\infty}\left(B_{r}\left(x_{0}\right), \mathbb{R}^{N}\right)$, it follows

$$
\begin{aligned}
& \left|\frac{1}{\left|B_{r}\left(x_{0}\right)\right|} \int_{B_{r}\left(x_{0}\right)} A\left(\nabla_{H} w, \nabla_{H} \phi\right) d x\right| \leq c_{1}\left[\Psi_{*}(2 r)+\Psi_{*}^{1 / 2}(2 r)\right. \\
& \left.\quad+\Psi_{*}^{1 / p}(2 r)+\vartheta\left(\Psi_{*}^{1 / 2}(2 r)\right)+\vartheta\left(\Psi_{*}^{1 / p}(2 r)\right)\right] \sup _{B_{r}\left(x_{0}\right)}\left|\nabla_{H} \phi\right|,
\end{aligned}
$$

where $c_{1}=C\left(p, M_{0}, \Lambda, C_{c}\right)$. Here, we say that $w$ is an approximately A-harmonic map. 
Proof. A direct calculation gives

$$
\begin{aligned}
& \frac{1}{\left|B_{r}\left(x_{0}\right)\right|} \int_{B_{r}\left(x_{0}\right)} \mathrm{A}\left(\nabla_{H} w, \nabla_{H} \phi\right) d x \\
& =\frac{1}{\left|B_{r}\left(x_{0}\right)\right|} \int_{B_{r}\left(x_{0}\right)} \int_{0}^{1}\left[\left(D_{P} A_{i}^{k}\left(\cdot, l\left(x_{0}^{\prime}\right), \nabla_{H} l\right)\right)_{x_{0}, r}\right. \\
& \left.\quad-\left(D_{P} A_{i}^{k}\left(\cdot l\left(x_{0}^{\prime}\right), \nabla_{H} l+s \nabla_{H} w\right)\right)_{x_{0}, r}\right] \nabla_{H} w \cdot \nabla_{H} \phi d s d x \\
& \quad+\frac{1}{\left|B_{r}\left(x_{0}\right)\right|} \int_{B_{r}\left(x_{0}\right)} \int_{0}^{1}\left(D_{P} A_{i}^{k}\left(\cdot, l\left(x_{0}^{\prime}\right), \nabla_{H} l+s \nabla_{H} w\right)\right)_{x_{0}, r} \nabla_{H} w \cdot \nabla_{H} \phi d s d x \\
& =: J_{1}+J_{2} .
\end{aligned}
$$

The treatment of $J_{1}$ is similar to that of Lemma 4.2 in [15]. In fact, we use (10), the monotonicity of $\vartheta$, Lemma 7 , Young's inequality, Jensen's inequality and Hölder's inequality to gain

$$
J_{1} \leq C\left(p, M_{0}, \Lambda\right)\left[\Phi(r)+\vartheta\left(\Phi^{1 / 2}(r)\right)+\vartheta\left(\Phi^{1 / p}(r)\right)\right] \sup _{B_{r}\left(x_{0}\right)}\left|\nabla_{H} \phi\right|
$$

Now, let us estimate $J_{2}$. Since

$$
\frac{1}{\left|B_{r}\left(x_{0}\right)\right|} \int_{B_{r}\left(x_{0}\right)}\left(A_{i}^{k}\left(\cdot, l\left(x_{0}^{\prime}\right), \nabla_{H} l\right)\right)_{x_{0}, r} \nabla_{H} \varphi d x=0
$$

we see

$$
\begin{aligned}
J_{2}= & \frac{1}{\left|B_{r}\left(x_{0}\right)\right|} \int_{B_{r}\left(x_{0}\right)}\left[\left(A_{i}^{k}\left(\cdot, l\left(x_{0}^{\prime}\right), \nabla_{H} u\right)\right)_{x_{0}, r}\right. \\
& \left.-\left(A_{i}^{k}\left(\cdot l\left(x_{0}^{\prime}\right), \nabla_{H} l\right)\right)_{x_{0}, r}\right] \nabla_{H} \phi d x \\
= & \frac{1}{\left|B_{r}\left(x_{0}\right)\right|} \int_{B_{r}\left(x_{0}\right)}\left(A_{i}^{k}\left(\cdot, l\left(x_{0}^{\prime}\right), \nabla_{H} u\right)\right)_{x_{0}, r} \nabla_{H} \phi d x .
\end{aligned}
$$

Noting from (17) that

$$
\begin{aligned}
& \frac{1}{\left|B_{r}\left(x_{0}\right)\right|} \int_{B_{r}\left(x_{0}\right)} A_{i}^{k}\left(x, u, \nabla_{H} u\right) \nabla_{H} \phi d x+\frac{1}{\left|B_{r}\left(x_{0}\right)\right|} \int_{B_{r}\left(x_{0}\right)} u \cdot T \phi d x \\
& -\frac{1}{\left|B_{r}\left(x_{0}\right)\right|} \int_{B_{r}\left(x_{0}\right)} B^{k}\left(x, u, \nabla_{H} u\right) \phi d x=0,
\end{aligned}
$$

$$
\begin{aligned}
J_{2}= & \frac{1}{\left|B_{r}\left(x_{0}\right)\right|} \int_{B_{r}\left(x_{0}\right)}\left(A_{i}^{k}\left(\cdot, l\left(x_{0}^{\prime}\right), \nabla_{H} u\right)\right)_{x_{0}, r} \nabla_{H} \phi d x \\
& -\frac{1}{\left|B_{r}\left(x_{0}\right)\right|} \int_{B_{r}\left(x_{0}\right)} A_{i}^{k}\left(x, u, \nabla_{H} u\right) \nabla_{H} \phi d x \\
& +\frac{1}{\left|B_{r}\left(x_{0}\right)\right|} \int_{B_{r}\left(x_{0}\right)} B^{k}\left(x, u, \nabla_{H} u\right) \phi d x-\frac{1}{\left|B_{r}\left(x_{0}\right)\right|} \int_{B_{r}\left(x_{0}\right)} u \cdot T \phi d x \\
= & \frac{1}{\left|B_{r}\left(x_{0}\right)\right|} \int_{B_{r}\left(x_{0}\right)}\left[\left(A_{i}^{k}\left(\cdot, l\left(x_{0}^{\prime}\right), \nabla_{H} u\right)\right)_{x_{0}, r}-A_{i}^{k}\left(x, l\left(x_{0}^{\prime}\right), \nabla_{H} u\right)\right] \nabla_{H} \phi d x \\
& +\frac{1}{\left|B_{r}\left(x_{0}\right)\right|} \int_{B_{r}\left(x_{0}\right)}\left[A_{i}^{k}\left(x, l\left(x_{0}^{\prime}\right), \nabla_{H} u\right)-A_{i}^{k}\left(x, \mathrm{u}, \nabla_{H} u\right)\right] \nabla_{H} \phi d x \\
& +\frac{1}{\left|B_{r}\left(x_{0}\right)\right|} \int_{B_{r}\left(x_{0}\right)} B^{k}\left(x, u, \nabla_{H} u\right) \phi d x-\frac{1}{\left|B_{r}\left(x_{0}\right)\right|} \int_{B_{r}\left(x_{0}\right)} u \cdot T \phi d x \\
= & J_{21}+J_{22}+J_{23}+J_{24} .
\end{aligned}
$$

The treatments of $J_{21}$ and $J_{22}$ are similar to that of Lemma 4.2 in [15]. To be specific, by (11), Lemma 7, Young's inequality and $\left|l\left(x_{0}^{\prime}\right)\right|+\left|\nabla_{H} l\right| \leq M_{0}$, one has

$$
J_{21} \leq C\left(p, M_{0}, \Lambda\right)[v(r)+\Phi(r)] \sup _{B_{r}\left(x_{0}\right)}\left|\nabla_{H} \phi\right|
$$

We use (9), Young's inequality, Jensen's inequality and Lemma 7 to get

$$
J_{22} \leq C\left(p, M_{0}, \Lambda\right)\left[\omega\left(\frac{1}{\left|B_{r}\left(x_{0}\right)\right|} \int_{B_{r}\left(x_{0}\right)}\left|u-l\left(x_{0}^{\prime}\right)\right|^{p} d x\right)+\Phi(r)\right] \sup _{B_{r}\left(x_{0}\right)}\left|\nabla_{H} \phi\right|
$$

It is worth noting that the treatments of $J_{23}$ and $J_{24}$ are different from that in [15]. Using the assumption (14), Hölder's inequality and Lemma 6, we obtain

$$
\begin{aligned}
J_{23} \leq & c \frac{1}{\left|B_{r}\left(x_{0}\right)\right|} \int_{B_{r}\left(x_{0}\right)}\left(\left|\nabla_{H} u\right|^{p}+|u|^{p^{*}+1}\right)^{1 /\left(p^{*}\right)^{\prime}} \cdot \phi d x \\
\leq & c r\left(\frac{1}{\left|B_{r}\left(x_{0}\right)\right|} \int_{B_{r}\left(x_{0}\right)}\left(\left|\nabla_{H} u\right|^{p}+|u|^{p^{*}}+1\right) d x\right)^{1 /\left(p^{*}\right)^{\prime}} \\
& \cdot\left(\frac{1}{\left|B_{r}\left(x_{0}\right)\right|} \int_{B_{r}\left(x_{0}\right)}\left(\frac{\phi}{r}\right)^{p^{*}} d x\right)^{1 / p^{*}} \\
\leq & c r\left(\frac{1}{\left|B_{r}\left(x_{0}\right)\right|} \int_{B_{r}\left(x_{0}\right)}\left(\left|\nabla_{H} u\right|^{p}+|u|^{p^{*}}+1\right) d x\right)^{1 /\left(p^{*}\right)^{\prime}} \\
& \cdot\left(\frac{1}{\left|B_{r}\left(x_{0}\right)\right|} \int_{B_{r}\left(x_{0}\right)}\left|\nabla_{H} \phi\right|^{p} d x\right)^{1 / p} \leq c \Psi_{*}^{1 / 2}(r) \sup _{B_{r}\left(x_{0}\right)}\left|\nabla_{H} \phi\right| .
\end{aligned}
$$

Noting

$$
T=X_{i} X_{n+i}-X_{n+i} X_{i}
$$

it implies 


$$
\begin{aligned}
J_{24}= & -\frac{1}{\left|B_{r}\left(x_{0}\right)\right|} \int_{B_{r}\left(x_{0}\right)}(u-l) \cdot T \phi d x \\
= & \frac{1}{\left|B_{r}\left(x_{0}\right)\right|} \int_{B_{r}\left(x_{0}\right)}(u-l) \cdot X_{n+i} X_{i} \phi d x \\
& -\frac{1}{\left|B_{r}\left(x_{0}\right)\right|} \int_{B_{r}\left(x_{0}\right)}(u-l) \cdot X_{i} X_{n+i} \phi d x \\
= & -\frac{1}{\left|B_{r}\left(x_{0}\right)\right|} \int_{B_{r}\left(x_{0}\right)} X_{n+i}(u-l) \cdot X_{i} \phi d x \\
& +\frac{1}{\left|B_{r}\left(x_{0}\right)\right|} \int_{B_{r}\left(x_{0}\right)} X_{i}(u-l) \cdot X_{n+i} \phi d x \\
\leq & \sup _{B_{r}\left(x_{0}\right)}\left|\nabla_{H} \phi\right| \frac{1}{\left|B_{r}\left(x_{0}\right)\right|} \int_{B_{r}\left(x_{0}\right)}\left|\nabla_{H}(u-l)\right| d x .
\end{aligned}
$$

In order to deal with $\left(1 /\left|B_{r}\left(x_{0}\right)\right|\right) \int_{B_{r}\left(x_{0}\right)}\left|\nabla_{H}(u-l)\right| d x$, we denote

$$
\begin{aligned}
& \Omega_{1}=\left\{\left|\nabla_{H} u-\nabla_{H} l\right| \leq 1\right\} \cap B_{r}\left(x_{0}\right), \\
& \Omega_{2}=\left\{\left|\nabla_{H} u-\nabla_{H} l\right|>1\right\} \cap B_{r}\left(x_{0}\right),
\end{aligned}
$$

so

$$
\begin{aligned}
& \frac{1}{\left|B_{r}\left(x_{0}\right)\right|} \int_{B_{r}\left(x_{0}\right)}\left|\nabla_{H}(u-l)\right| d x=\frac{1}{\left|B_{r}\left(x_{0}\right)\right|} \int_{\Omega_{1}}\left|\nabla_{H}(u-l)\right| d x \\
& +\frac{1}{\left|B_{r}\left(x_{0}\right)\right|} \int_{\Omega_{2}}\left|\nabla_{H}(u-l)\right| d x \leq\left(\frac{\left|\Omega_{1}\right|}{\left|B_{r}\left(x_{0}\right)\right|}\right)^{1 / 2} \\
& \cdot\left(\frac{1}{\left|B_{r}\left(x_{0}\right)\right|} \int_{\Omega_{1}}\left|\nabla_{H}(u-l)\right|^{2} d x\right)^{1 / 2}+\left(\frac{\left|\Omega_{2}\right|}{\left|B_{r}\left(x_{0}\right)\right|}\right)^{1 / p^{\prime}} \\
& \cdot\left(\frac{1}{\left|B_{r}\left(x_{0}\right)\right|} \int_{\Omega_{2}}\left|\nabla_{H}(u-l)\right|^{p} d x\right)^{1 / p} \\
& \quad \leq\left(\frac{1}{\left|B_{r}\left(x_{0}\right)\right|} \int_{\Omega_{1}}\left|\nabla_{H}(u-l)\right|^{2} d x\right)^{1 / 2} \\
& +\left(\frac{1}{\left|B_{r}\left(x_{0}\right)\right|} \int_{\Omega_{2}}\left|\nabla_{H}(u-l)\right|^{p} d x\right)^{1 / p} \leq c\left(\Phi^{1 / 2}(r)+\Phi^{1 / p}(r)\right) .
\end{aligned}
$$

Then,

$$
J_{24} \leq c\left(\Phi^{1 / 2}(r)+\Phi^{1 / p}(r)\right) \sup _{B_{r}\left(x_{0}\right)}\left|\nabla_{H} \phi\right| .
$$

Now, we replace (80)-(87) in (79) to see

$$
\begin{aligned}
J_{2} \leq & C\left(p, M_{0}, \Lambda\right)\left[\Phi(r)+\Phi^{1 / 2}(r)+\Phi^{1 / p}(r)\right. \\
& \left.+\omega\left(\frac{1}{\left|B_{r}\left(x_{0}\right)\right|} \int_{B_{r}\left(x_{0}\right)}\left|u-l\left(x_{0}^{\prime}\right)\right|^{p} d x\right)\right] \sup _{B_{r}\left(x_{0}\right)}\left|\nabla_{H} \phi\right| \\
& +C\left(p, M_{0}, \Lambda\right)\left[v(r)+\Psi_{*}^{1 / 2}(r)\right] \sup _{B_{r}\left(x_{0}\right)}\left|\nabla_{H} \phi\right| \leq C\left(p, M_{0}, \Lambda\right) \\
& \cdot\left[\Phi(r)+\Phi^{1 / 2}(r)+\Phi^{1 / p}(r)+\Psi_{*}(r)+\Psi_{*}^{1 / 2}(r)\right] \sup _{B_{r}\left(x_{0}\right)}\left|\nabla_{H} \phi\right| .
\end{aligned}
$$

Finally, we substitute (75) and (88) into (74) and then use Lemma 11 to get

$$
\begin{aligned}
& \left|\frac{1}{\left|B_{r}\left(x_{0}\right)\right|} \int_{B_{r}\left(x_{0}\right)} \mathrm{A}\left(\nabla_{H} w, \nabla_{H} \phi\right) d x\right| \\
& \leq C\left(p, M_{0}, \Lambda\right)\left[\Phi(r)+\Phi^{1 / 2}(r)+\Phi^{1 / p}(r)+\Psi_{*}(r)\right. \\
& \left.\quad+\Psi_{*}^{1 / 2}(r)+\vartheta\left(\Phi^{1 / 2}(r)\right)+\vartheta\left(\Phi^{1 / p}(r)\right)\right] \sup _{B_{r}\left(x_{0}\right)}\left|\nabla_{H} \phi\right| \\
& \leq C\left(p, M_{0}, \Lambda, C_{c}\right)\left[\Psi_{*}(2 r)+\Psi_{*}^{1 / 2}(2 r)+\Psi_{*}^{1 / p}(2 r)\right. \\
& \left.\quad+\vartheta\left(\Psi_{*}^{1 / 2}(2 r)\right)+\vartheta\left(\Psi_{*}^{1 / p}(2 r)\right)\right] \sup _{B_{r}\left(x_{0}\right)}\left|\nabla_{H} \phi\right|,
\end{aligned}
$$

i.e., (73) holds.

Lemma 13 (decay estimate). Assume the assumptions of Theorem 1 are satisfied and $B_{r}\left(x_{0}\right) \subset \Omega$ with $r \leq r_{0}$. For constants $\theta \in(0,1 / 4], \delta=\delta(\wp, N, p, v, L, \theta) \in(0,1]$ and $\rho \in(0,1]$ from the A-harmonic approximation Lemma 10, we impose the following smallness conditions:

(i) $\Psi_{*}^{1 / 2}(r)<\delta / 2$;

(ii) $\rho:=\sqrt{\Psi_{*}(r)+(\delta / 2)^{-2}\left[\Psi_{*}^{1 / 2}(r)+\Psi_{*}^{1 / p}(r)+\vartheta\left(\Psi_{*}^{1 / 2}(r)\right)+\vartheta\left(\Psi_{*}^{1 / p}(r)\right)\right]^{2}} \leq 1$.

Then, it holds

$$
\Psi\left(x_{0}, \theta r, l_{x_{0}, \theta r}\right) \leq c_{3} \theta^{2} \Psi_{*}\left(x_{0}, r, l_{x_{0}, r}\right),
$$

where $l_{x_{0}, \theta r}$ and $l_{x_{0}, r}$ denote the minimizing horizontal affine functions with $\left|l_{x_{0}, \theta r}\left(x_{0}^{\prime}\right)\right|+\left|\nabla_{H} l_{x_{0}, \theta r}\right| \leq M_{0}$ and $\left|l_{x_{0}, r}\left(x_{0}^{\prime}{ }_{0}\right)\right|+$ $\left|\nabla_{H} l_{x_{0}, r}\right| \leq M_{0}$, respectively, and the constant $c_{3}$ depends only on $n, N, p, \lambda, \Lambda, \delta$.

Proof. We divide several steps to prove (90).

Step 1. Let us take

$$
\tilde{w}=\frac{u-l_{x_{0}, r}}{c_{2}}
$$

where $l_{x_{0}, r}=u_{x_{0}, r}+\nabla_{H} l_{x_{0}, r}\left(x^{\prime}-x_{0}^{\prime}\right), c_{2}=\max \left\{c_{1}, \sqrt{C_{c}}\right\}$. We first claim that $\tilde{w}$ satisfies the assumptions (51) and (52) Lemma 10.

In fact, for $l=l_{x_{0}, r}$ and any $\phi \in C_{0}^{\infty}\left(B_{r}\left(x_{0}\right), \mathbb{R}^{N}\right)$, we have by using Lemma 12, Lemma 7 (2), and assumptions (ii) and (i) that

$$
\begin{aligned}
& \left|\frac{1}{\left|B_{r / 2}\left(x_{0}\right)\right|} \int_{B_{r 2}\left(x_{0}\right)} \mathrm{A}\left(\nabla_{H} \tilde{w}, \nabla_{H} \phi\right) d x\right| \\
& \quad \leq \rho \frac{c_{1}}{c_{2}}\left[\frac{\Psi_{*}(r)+\Psi_{*}^{1 / 2}(r)+\Psi_{*}^{1 / p}(r)+\vartheta\left(\Psi_{*}^{1 / 2}(r)\right)+\vartheta\left(\Psi_{*}^{1 / p}(r)\right)}{\rho}\right] \sup _{B_{r / 2}\left(x_{0}\right)}\left|\nabla_{H} \phi\right| \\
& \quad \leq \rho\left[\Psi_{*}^{1 / 2}(r)+\frac{\delta}{2}\right]_{B_{r / 2}\left(x_{0}\right)}\left|\nabla_{H} \phi\right| \leq \rho \delta \sup _{B_{r 2}\left(x_{0}\right)}\left|\nabla_{H} \phi\right| .
\end{aligned}
$$


Now, it deduces from Lemma 7 (2) and Lemma 11 that

$$
\begin{aligned}
& \frac{1}{\left|B_{r / 2}\left(x_{0}\right)\right|} \int_{B_{r / 2}\left(x_{0}\right)}\left|V\left(\nabla_{H} \tilde{w}\right)\right|^{2} d x \\
& \leq \frac{1}{c_{2}^{2}} \frac{1}{\left|B_{r / 2}\left(\mathrm{x}_{0}\right)\right|} \int_{B_{r / 2}\left(x_{0}\right)}\left|V\left(\nabla_{H} u-\nabla_{H} l_{x_{0}, r}\right)\right|^{2} d x \\
& \quad=\frac{\Phi\left(r / 2, l_{x_{0}, r}\right)}{c_{2}^{2}} \leq \frac{C_{c} \Psi_{*}(r)}{c_{2}^{2}} \leq \rho^{2} .
\end{aligned}
$$

Then, the assumptions (51) and (52) of Lemma 10 are satisfied by (92) and (93).

Using Lemma 10, it follows that there exists an $A$-harmonic function $h \in C^{\infty}\left(\mathrm{B}_{r / 2}\left(x_{0}\right), \mathbb{R}^{N}\right)$ satisfies

$$
\frac{1}{\left|B_{r / 2}\left(x_{0}\right)\right|} \int_{B_{r / 2}\left(x_{0}\right)}\left|V\left(\frac{\tilde{w}-\rho h}{r}\right)\right|^{2} d x \leq \rho^{2} \varepsilon
$$

and

$$
\frac{1}{\left|B_{r / 2}\left(x_{0}\right)\right|} \int_{B_{r / 2}\left(x_{0}\right)}\left|V\left(\nabla_{H} h\right)\right|^{2} d x \leq 1
$$

Step 2. We estimate $\left(1 /\left|B_{\theta r}\left(x_{0}\right)\right|\right) \int_{B_{\theta r}\left(x_{0}\right)}\left|V\left(u-l_{x_{0}, \theta r} / \theta r\right)\right|^{2} d x$.

Let us denote

$$
l^{h}(x)=h_{x_{0}, \theta r}+\left(\nabla_{H} h\right)_{x_{0}, \theta r}\left(x^{\prime}-x_{0}^{\prime}\right)
$$

and compute by (3) and (2) of Lemma 7, Lemma 8 and (94) that

$$
\begin{aligned}
& \frac{1}{\left|B_{\theta r}\left(x_{0}\right)\right|} \int_{B_{\theta r}\left(x_{0}\right)}\left|V\left(\frac{\tilde{w}-\rho l^{h}}{\theta r}\right)\right|^{2} d x \\
& =\frac{1}{\left|B_{\theta r}\left(x_{0}\right)\right|} \int_{B_{\theta r}\left(x_{0}\right)}\left|V\left(\frac{\tilde{w}-\rho h+\rho\left(h-h_{x_{0}, \theta r}-\left(\nabla_{H} h\right)_{x_{0}, \theta r}\left(\mathrm{x}^{\prime}-x_{0}^{\prime}\right)\right)}{\theta r}\right)\right|^{2} d x \\
& \leq c_{p} \frac{1}{\left|B_{\theta r}\left(x_{0}\right)\right|} \int_{B_{\theta r}\left(x_{0}\right)}\left|V\left(\frac{\tilde{w}-\rho h}{\theta r}\right)\right|^{2} d x \\
& \quad+c_{p} \frac{1}{\left|B_{\theta r}\left(x_{0}\right)\right|} \int_{B_{\theta r}\left(x_{0}\right)}\left|\rho V\left(\frac{h-h_{x_{0}, \theta r}-\left(\nabla_{H} h\right)_{x_{0}, \theta r}\left(x^{\prime}-x_{0}^{\prime}\right)}{\theta r}\right)\right|^{2} d x \\
& \leq c_{p} \theta^{-2}(2 \theta)^{-\wp} \frac{1}{\left|B_{r / 2}\left(x_{0}\right)\right|} \int_{B_{r / 2}\left(x_{0}\right)}\left|V\left(\frac{\tilde{w}-\rho h}{r}\right)\right|^{2} d x \\
& \quad+c_{p} C_{p} \rho^{2} \frac{1}{\left|B_{\theta r}\left(x_{0}\right)\right|} \int_{B_{\theta r}\left(x_{0}\right)}\left|V\left(\nabla_{H} h-\left(\nabla_{H} h\right)_{x_{0}, \theta r}\right)\right|^{2} d x \\
& \leq c_{p}\left[\theta^{-2}(2 \theta)^{-\wp} \rho^{2} \varepsilon+C_{p}^{2} \rho^{2}(\theta r)^{2} \frac{1}{\left|B_{\theta r}\left(x_{0}\right)\right|} \int_{B_{\theta r}\left(x_{0}\right)}\left|V\left(\nabla_{H}^{2} h\right)\right|^{2} d x\right] .
\end{aligned}
$$

In order to estimate (97), we need to deal with $\left(1 / \mid B_{\theta r}\right.$ $\left.\left(x_{0}\right) \mid\right) \int_{B_{\theta r}\left(x_{0}\right)}\left|\mathrm{V}\left(\nabla_{H}^{2} h\right)\right|^{2} d x$. Noting Lemma 9, it derives $h \epsilon$ $C^{\infty}\left(\Omega, \mathbb{R}^{N}\right)$ and

$$
\sup _{B_{r / 2}\left(x_{0}\right)}\left(\left|\nabla_{H} h\right|^{2}+\left|\nabla_{H}^{2} h\right|^{2}\right) \leq c r^{-2} \frac{1}{\left|B_{r}\left(x_{0}\right)\right|} \int_{B_{r}\left(x_{0}\right)}\left|\nabla_{H} h\right|^{2} d x,
$$

so we have

$$
\begin{gathered}
\sup _{B_{r / 2}\left(x_{0}\right)}\left|\nabla_{H}^{2} h\right|^{2} \leq c r^{-2} \frac{1}{\left|B_{r}\left(x_{0}\right)\right|} \int_{B_{r}\left(x_{0}\right)}\left|\nabla_{H} h\right|^{2} d x, \\
\left|\nabla_{H} h\right| \leq\left(c r^{-2} \frac{1}{\left|B_{r}\left(x_{0}\right)\right|} \int_{B_{r}\left(x_{0}\right)}\left|\nabla_{H} h\right|^{2} d x\right)^{1 / 2}:=M \text { in } B_{r / 2}\left(x_{0}\right) .
\end{gathered}
$$

Hence, for $\theta \in(0,1 / 4]$, we use Lemma 7 (1), (99), (100), Hölder's inequality and (95) to obtain

$$
\begin{aligned}
& \frac{1}{\left|B_{\theta r}\left(x_{0}\right)\right|} \int_{B_{\theta r}\left(x_{0}\right)}\left|V\left(\nabla_{H}^{2} h\right)\right|^{2} d x \leq \frac{1}{\left|B_{\theta r}\left(x_{0}\right)\right|} \int_{B_{\theta r}\left(x_{0}\right)}\left|\nabla_{H}^{2} h\right|^{2} d x \\
& \leq \sup _{B_{r / 4}\left(x_{0}\right)}\left|\nabla_{H}^{2} h\right|^{2} \leq c r^{-2} \frac{1}{\left|B_{r / 2}\left(x_{0}\right)\right|} \int_{B_{r / 2}\left(x_{0}\right)}\left|\nabla_{H} h\right|^{2} d x \\
& \leq c r^{-2} M \frac{1}{\left|B_{r / 2}\left(x_{0}\right)\right|} \int_{B_{r / 2}\left(x_{0}\right)}\left|\nabla_{H} h\right| d x \\
& =c r^{-2} M\left[\frac{1}{\left|B_{r / 2}\left(x_{0}\right)\right|} \int_{B_{r / 2}\left(x_{0}\right) \cap\left\{\left|\nabla_{H} h\right| \leq 1\right\}}\left|\nabla_{H} h\right| d x\right. \\
& \left.+\frac{1}{\left|B_{r / 2}\left(x_{0}\right)\right|} \int_{B_{r / 2}\left(x_{0}\right) \cap\left\{\left|\nabla_{H} h\right|>1\right\}}\left|\nabla_{H} h\right| d x\right] \\
& \leq c r^{-2} M\left[\left(\frac{1}{\left|B_{r / 2}\left(x_{0}\right)\right|} \int_{B_{r / 2}\left(x_{0}\right) \cap\left\{\left|\nabla_{H} h\right| \leq 1\right\}}\left|\nabla_{H} h\right|^{2} d x\right)^{1 / 2}\right. \\
& \left.+\left(\frac{1}{\left|B_{r / 2}\left(x_{0}\right)\right|} \int_{B_{r / 2}\left(x_{0}\right) \cap\left\{\left|\nabla_{H} h\right|>1\right\}}\left|\nabla_{H} h\right|^{p} d x\right)^{1 / p}\right] \\
& \leq 2 c r^{-2} M\left[\left(\frac{1}{\left|B_{r / 2}\left(x_{0}\right)\right|} \int_{B_{r / 2}\left(x_{0}\right)}\left|V\left(\nabla_{H} h\right)\right|^{2} d x\right)^{1 / 2}\right. \\
& \left.+\left(\frac{1}{\left|B_{r / 2}\left(x_{0}\right)\right|} \int_{B_{r / 2}\left(x_{0}\right)}\left|V\left(\nabla_{H} h\right)\right|^{2} d x\right)^{1 / p}\right] \leq \mathrm{Cr}^{-2} \text {. }
\end{aligned}
$$

By substituting (101) into (97), we get

$$
\begin{aligned}
& \frac{1}{\left|B_{\theta r}\left(x_{0}\right)\right|} \int_{B_{\theta r}\left(x_{0}\right)}\left|V\left(\frac{\tilde{w}-\rho l^{h}}{\theta r}\right)\right|^{2} d x \\
& \quad \leq c_{p}\left[\theta^{-2}(2 \theta)^{-\wp} \rho^{2} \varepsilon+C C_{p}^{2} \rho^{2}(\theta r)^{2} r^{-2}\right] \\
& \quad \leq c\left(p, C_{p}, C\right) \rho^{2}\left[\theta^{-2-\wp} \varepsilon+\theta^{2}\right] .
\end{aligned}
$$

Since $\varepsilon>0$ in (102) is arbitrary, we take especially $\varepsilon=\theta^{\wp+4}$. Noting

$$
\Psi_{*}(r)<1 \text { and } \frac{2}{p}>1 \text {, }
$$

we use (ii) and the monotonicity of $\vartheta$ to obtain 


$$
\begin{aligned}
\rho^{2}= & \Psi_{*}(r)+\left(\frac{\delta}{2}\right)^{-2}\left[\Psi_{*}^{1 / 2}(r)+\Psi_{*}^{1 / p}(r)+\vartheta\left(\Psi_{*}^{1 / 2}(r)\right)\right. \\
& \left.+\vartheta\left(\Psi_{*}^{1 / p}(r)\right)\right]^{2} \leq \Psi_{*}(r)+16 \delta^{-2}\left(\Psi_{*}^{1 / 2}(r)+\Psi_{*}^{1 / p}(r)\right)^{2} \\
\leq & \Psi_{*}(r)+64 \delta^{-2} \Psi_{*}(r) .
\end{aligned}
$$

Therefore, it shows from (102) that

$\frac{1}{\left|B_{\theta r}\left(x_{0}\right)\right|} \int_{B_{\theta r}\left(x_{0}\right)}\left|V\left(\frac{\tilde{w}-\rho l^{h}}{\theta r}\right)\right|^{2} d x \leq c\left(p, C_{p}, C\right) \theta^{2}\left(1+64 \delta^{-2}\right) \Psi_{*}(r)$.

Now, we substitute $\tilde{w}=\left(u-l_{x_{0}, r}\right) / c_{2}$ into (105) and use Lemma 7 (2) to gain

$$
\begin{aligned}
& \frac{1}{\left|B_{\theta r}\left(x_{0}\right)\right|} \int_{B_{\theta r}\left(x_{0}\right)}\left|V\left(\frac{u-l_{x_{0}, r}-c_{2} \rho l^{h}}{\theta r}\right)\right|^{2} d x \\
& \quad \leq c_{2}^{2} c\left(p, C_{p}, C\right) \theta^{2}\left(1+64 \delta^{-2}\right) \Psi_{*}(r) .
\end{aligned}
$$

Since $l_{x_{0}, \theta r}$ is a minimizer of $\left(1 /\left|B_{\theta r}\left(x_{0}\right)\right|\right) \int_{B_{\theta r}\left(x_{0}\right)}$ $|u-l|^{2} d x$, we have

$$
\begin{aligned}
& \frac{1}{\left|B_{\theta r}\left(x_{0}\right)\right|} \int_{B_{\theta r}\left(x_{0}\right)}\left|V\left(\frac{u-l_{x_{0}, \theta r}}{\theta r}\right)\right|^{2} d x \\
& \quad \leq \frac{1}{\left|B_{\theta r}\left(x_{0}\right)\right|} \int_{B_{\theta r}\left(x_{0}\right)}\left|V\left(\frac{\mathrm{u}-l_{x_{0}, r}-c_{2} \rho l^{h}}{\theta r}\right)\right|^{2} d x \\
& \leq c_{2}^{2} c\left(p, C_{p}, C\right) \theta^{2}\left(1+64 \delta^{-2}\right) \Psi_{*}(r) \leq c_{3} \theta^{2} \Psi_{*}(r) .
\end{aligned}
$$

Step 3. We estimate $\left(1 /\left|B_{\theta r}\left(x_{0}\right)\right|\right) \int_{B_{\theta r}\left(x_{0}\right)}\left|\left(u-l_{x_{0}, \theta r}\right) / \theta r\right|^{2} d x$.

To do so, let us first deal with $\left(1 /\left|B_{\theta r}\left(x_{0}\right)\right|\right) \int_{B_{\theta r}\left(x_{0}\right)}$ $\left|\left(\tilde{w}-\rho l^{h}\right) / \theta r\right|^{2} d x$. Since $|V((\tilde{w}-\rho h) / r)|$ is bounded almost everywhere by (94), we denote its upper bound by $M_{1}$. It implies by Lemma 7 (1) that

(1) when $|(\tilde{w}-\rho h) / r| \leq 1$, it follows

$$
\left|\frac{\tilde{w}-\rho h}{r}\right| \leq \sqrt{2}\left|V\left(\frac{\tilde{w}-\rho h}{r}\right)\right| \leq \sqrt{2} M_{1}
$$

(2) when $|(\tilde{w}-\rho h) / r|>1$, we have

$\left|\frac{\tilde{w}-\rho h}{r}\right| \leq\left(\sqrt{2}\left|V\left(\frac{\tilde{w}-\rho h}{r}\right)\right|\right)^{2 / p} \leq\left(\sqrt{2} M_{1}\right)^{2 / p}$

Hence,

$$
\left|\frac{\tilde{w}-\rho h}{r}\right| \leq \max \left\{\sqrt{2} M_{1},\left(\sqrt{2} M_{1}\right)^{\frac{2}{p}}\right\}:=M_{2} \text {. }
$$

Now, by using

$$
l^{h}(x)=h_{x_{0}, \theta r}+\nabla_{H} h_{x_{0}, \theta r}\left(x^{\prime}-x_{0}^{\prime}\right),
$$

Lemma 8, (101), Lemma 7 (1) and (2), (94) and the similar proof to (102), we get

$$
\begin{aligned}
& \frac{1}{\left|B_{\theta r}\left(x_{0}\right)\right|} \int_{B_{\theta r}\left(x_{0}\right)}\left|\frac{\tilde{w}-\rho l^{h}}{\theta r}\right|^{2} d x \\
& =\frac{1}{\left|B_{\theta r}\left(x_{0}\right)\right|} \int_{B_{\theta r}\left(x_{0}\right)}\left|\frac{\tilde{w}-\rho l^{h}+\rho\left(h-h_{x_{0}, \theta r}-\left(\nabla_{H} h\right)_{x_{0}, \theta r}\left(x^{\prime}-x_{0}^{\prime}\right)\right)}{\theta r}\right|^{2} d x \\
& \leq 2\left[\frac{1}{\left|B_{\theta r}\left(x_{0}\right)\right|} \int_{B_{\theta r}\left(x_{0}\right)}\left|\frac{\tilde{w}-\rho h}{\theta r}\right|^{2} d x\right. \\
& \left.+\rho^{2} \frac{1}{\left|B_{\theta r}\left(x_{0}\right)\right|} \int_{B_{\theta r}\left(x_{0}\right)}\left|\frac{h-h_{x_{0}, \theta r}-\left(\nabla_{H} h\right)_{x_{0}, \theta r}\left(x^{\prime}-x_{0}^{\prime}\right)}{\theta r}\right|^{2} d x\right] \\
& \leq 2\left[\theta^{-2}(2 \theta)^{-r} \frac{1}{\left|B_{r / 2}\left(x_{0}\right)\right|} \int_{B_{r / 2}\left(x_{0}\right)}\left|\frac{\tilde{w}-\rho h}{r}\right|^{2} d x\right. \\
& \left.+C_{p} \rho^{2} \frac{1}{\left|B_{\theta r}\left(x_{0}\right)\right|} \int_{B_{\theta r}\left(x_{0}\right)}\left|\nabla_{H} h-\left(\nabla_{H} h\right)_{x_{0}, \theta r}\right|^{2} d x\right] \\
& \leq 2\left[2^{-\xi} M_{2}^{2} \theta^{-2-\boldsymbol{\gamma}} \frac{1}{\left|B_{r / 2}\left(x_{0}\right)\right|} \int_{B_{r / 2}\left(x_{0}\right)}\left|\frac{\tilde{w}-\rho h}{2 M_{2} r}\right|^{2} d x\right. \\
& \left.+C_{p}^{2} \rho^{2}(\theta r)^{2} \frac{1}{\left|B_{\theta r}\left(x_{0}\right)\right|} \int_{B_{\theta r}\left(x_{0}\right)}\left|\nabla_{H}^{2} h\right|^{2} d x\right] \\
& \leq 2\left[2^{1-\beta} M_{2}^{2} \theta^{-2-\beta} \frac{1}{\left|B_{r / 2}\left(x_{0}\right)\right|} \int_{B_{r / 2}\left(x_{0}\right)}\left|V\left(\frac{\tilde{w}-\rho h}{2 M_{2} r}\right)\right|^{2} d x\right. \\
& \left.+C C_{p}^{2} \rho^{2}(\theta r)^{2} r^{-2}\right] \leq 2\left[2^{-\wp} \theta^{-2-\wp} \frac{1}{\left|B_{r / 2}\left(x_{0}\right)\right|} \int_{B_{r / 2}\left(x_{0}\right)}\left|V\left(\frac{\tilde{w}-\rho h}{r}\right)\right|^{2} d x\right. \\
& \left.+C C_{p}^{2} \rho^{2}(\theta r)^{2} r^{-2}\right] \leq 2\left[\theta^{-2-\beta} 2^{-\gamma} \rho^{2} \varepsilon+C C_{p}^{2} \rho^{2}(\theta r)^{2} r^{-2}\right] \\
& \leq c\left(p, C_{p}, C\right) \rho^{2}\left[\theta^{-2-\beta} \varepsilon+\theta^{2}\right] \leq c\left(p, C_{p}, C\right) \theta^{2}\left(1+64 \delta^{-2}\right) \Psi_{*}(r) \text {. }
\end{aligned}
$$

We substitute $\tilde{w}=\left(u-l_{x_{0}, r}\right) / c_{2}$ into the inequality above to obtain

$$
\begin{aligned}
& \frac{1}{\left|B_{\theta r}\left(x_{0}\right)\right|} \int_{B_{\theta r}\left(x_{0}\right)}\left|\frac{u-l_{x_{0}, r}-c_{2} \rho l^{h}}{\theta r}\right|^{2} d x \\
& \quad \leq c_{2}^{2} c\left(p, C_{p}, C\right) \theta^{2}\left(1+64 \delta^{-2}\right) \Psi_{*}(r),
\end{aligned}
$$

and know from that $l_{x_{0}, \theta r}$ is a minimizer of $\left(1 /\left|B_{\theta r}\left(x_{0}\right)\right|\right)$ $\int_{B_{\theta r}\left(x_{0}\right)}|u-l|^{2} d x$ that

$$
\begin{aligned}
& \frac{1}{\left|B_{\theta r}\left(x_{0}\right)\right|} \int_{B_{\theta r}\left(x_{0}\right)}\left|\frac{u-l_{x_{0}, \theta r}}{\theta r}\right|^{2} d x \\
& \quad \leq c_{2}^{2} c\left(p, C_{p}, C\right) \theta^{2}\left(1+64 \delta^{-2}\right) \Psi_{*}(r) \leq c_{3} \theta^{2} \Psi_{*}(r) .
\end{aligned}
$$


Step 4. Combining (107) with (114), we derive (90). Lemma 13 is proved.

Before stating a new lemma, we introduce Campanatotype functions. For the fixed Hölder exponent $\alpha \in(0,1)$, define a Campanato-type function by

$$
Y_{\alpha}\left(x_{0}, r\right)=r^{-p \alpha} \frac{1}{\left|B_{r}\left(x_{0}\right)\right|} \int_{B_{r}\left(x_{0}\right)}\left|u-u_{x_{0}, r}\right|^{p} d x, 1<p<2 .
$$

We can prove the following lemma from Lemma 13.

Lemma 14 (iteration relations). Assume the assumptions of Theorem 1 are satisfied. For any $\alpha \in(0,1)$, there exist constants $\varepsilon_{*}, \kappa_{*}, r_{*}$ and $\theta \in(0,1 / 8]$, such that if for $0<r<r_{*}$ with $B_{r}\left(x_{0}\right) \subset \Omega$, one has

$$
\Psi\left(x_{0}, r, l_{x_{0}, r}\right)<\varepsilon_{*} \text { and } Y_{\alpha}\left(x_{0}, r\right)<\kappa_{*},
$$

then, for any $k \in \mathbb{N}$, it holds

$$
\Psi\left(x_{0}, \theta^{k} r, l_{x_{0}, \theta^{k} r}\right)<\epsilon_{*} \text { and } Y_{\alpha}\left(x_{0}, \theta^{k} r\right)<\kappa_{*} .
$$

Proof. Its proof is similar to Lemma 4.4 in [15]. Actually, $\Psi_{*}\left(\theta^{k} r\right) \leq \varepsilon_{*}$ has been proved in [15], so we only need to take $\varepsilon_{*} \leq \min \left\{\left(\theta^{\wp+p \rho} / 8\right)^{2 / p}, \delta^{2} /\left(4 \delta^{2}+256\right)\right\}$ and change the estimate of $\rho\left(\theta^{k} r\right)$ in [15] to

$$
\begin{aligned}
\rho\left(\theta^{k} r\right) & :=\sqrt{\Psi_{*}\left(\theta^{k} r\right)+\left(\frac{\delta}{2}\right)^{-2}\left[\Psi_{*}^{1 / 2}\left(\theta^{k} r\right)+\Psi_{*}^{1 / p}\left(\theta^{k} r\right)+\vartheta\left(\Psi_{*}^{1 / 2}\left(\theta^{k} r\right)\right)+\vartheta\left(\Psi_{*}^{1 / p}\left(\theta^{k} r\right)\right]^{2}\right.} \\
& \leq \sqrt{4 \varepsilon_{*}+16 \delta^{-2}\left[\sqrt{4 \varepsilon_{*}}+\sqrt[p]{4 \varepsilon_{*}}\right]^{2}} \leq \sqrt{4 \varepsilon_{*}+16 \delta^{-2}\left[2 \sqrt{4 \varepsilon_{*}}\right]^{2}}=\sqrt{\left(\frac{4 \delta^{2}+256}{\delta^{2}}\right) \varepsilon_{*}} \leq 1 .
\end{aligned}
$$

\section{Proof of Theorem 1}

Proof of Theorem 1 is finished with two steps.

Step 1 . We prove $u \in \mathrm{C}_{\text {loc }}^{0, \alpha}\left(\Omega \backslash \Omega_{0}, \mathbb{R}^{N}\right)$. In fact, by Lebesgue's differentiation theorem ([27]), we get $\left|\sum_{1} \cup \sum_{2}\right|=0$, so our aim is to show that $u$ is Hölder continuous for every $x_{0} \in$ $\Omega \backslash\left(\sum_{1} \cup \sum_{2}\right)$. For any $0<r_{0}<\operatorname{dist}\left(x_{0}, \partial \Omega\right)$, we use Lemma 8 , (43) and Lemma 7 (1) to gain

$$
\begin{aligned}
\Psi\left(x_{0}, r_{0}, l_{x_{0}, r_{0}}\right)= & \frac{1}{\left|B_{r_{0}}\left(x_{0}\right)\right|} \int_{B_{r_{0}}\left(x_{0}\right)}\left|V\left(\frac{u-l_{x_{0}, r_{0}}}{r_{0}}\right)\right|^{2} d x \\
& +\frac{1}{\left|B_{r_{0}}\left(x_{0}\right)\right|} \int_{B_{r_{0}}\left(x_{0}\right)}\left|\frac{u-l_{x_{0}, r_{0}}}{r_{0}}\right|^{2} d x \\
\leq & c_{p}^{2}\left[\frac{1}{\left|B_{r_{0}}\left(x_{0}\right)\right|} \int_{B_{r_{0}}\left(x_{0}\right)}\left|V\left(\nabla_{H} u-\nabla_{H} l_{x_{0}, r_{0}}\right)\right|^{2} d x\right. \\
& \left.+\frac{1}{\left|B_{r_{0}}\left(x_{0}\right)\right|} \int_{B_{r_{0}}\left(x_{0}\right)}\left|\nabla_{H} u-\nabla_{H} l_{x_{0}, r_{0}}\right|^{2} d x\right] \\
\leq & 2 c_{p}^{2} \frac{1}{\left|B_{r_{0}}\left(x_{0}\right)\right|} \int_{B_{r_{0}}\left(x_{0}\right)}\left|\nabla_{H} u-\nabla_{H} l_{x_{0}, r_{0}}\right|^{2} d x .
\end{aligned}
$$

For any $\alpha \in(0,1)$ and $r_{0} \leq 1$, using

$$
l_{x_{0}, r_{0}}=u_{x_{0}, r_{0}}+\nabla_{H} l_{x_{0}, r_{0}}\left(x^{\prime}-x_{0}^{\prime}\right)
$$

(from it, one sees $u_{x_{0}, r_{0}}=l_{x_{0}, r_{0}}-\nabla_{H} l_{x_{0}, r_{0}}\left(x^{\prime}-x_{0}^{\prime}\right)$ ), $\mid l_{x_{0}, r_{0}}$ $|+| \nabla_{H} l_{x_{0}, r_{0}} \mid \leq M_{0}$ and Hölder's inequality, it infers

$$
\begin{aligned}
Y_{\alpha}\left(x_{0}, r_{0}\right) & =r_{0}^{-p \alpha} \frac{1}{\left|B_{r_{0}}\left(x_{0}\right)\right|} \int_{B_{r_{0}}\left(x_{0}\right)}\left|u-u_{x_{0}, r_{0}}\right|^{p} d x \\
& =r_{0}^{p-p \alpha} \frac{1}{\left|B_{r_{0}}\left(x_{0}\right)\right|} \int_{B_{r_{0}}\left(x_{0}\right)}\left|\frac{u-u_{x_{0}, r_{0}}}{r_{0}}\right|^{p} d x \\
& \leq r_{0}^{p-p \alpha}\left[\frac{1}{\left|B_{r_{0}}\left(x_{0}\right)\right|} \int_{B_{r_{0}}\left(x_{0}\right)}\left(\left|\frac{u-l_{x_{0}, r_{0}}}{r_{0}}\right|^{p}+\left|\nabla_{H} l_{x_{0}, r_{0}}\right|\right)^{p} d x\right] \\
& \leq C_{p} r_{0}^{p-p \alpha}\left[\frac{1}{\left|B_{r_{0}}\left(x_{0}\right)\right|} \int_{B_{r_{0}}\left(x_{0}\right)}\left|\frac{u-l_{x_{0}, r_{0}}}{r_{0}}\right|^{p} d x+\left|\nabla_{H} l_{x_{0}, r_{0}}\right|^{p]}\right] \\
& \leq C\left(p, M_{0}\right) r_{0}^{p-p \alpha}\left[\left(\frac{1}{\left|B_{r_{0}}\left(x_{0}\right)\right|} \int_{B_{r_{0}}\left(x_{0}\right)}\left|\frac{u-l_{x_{0}, r_{0}}}{r_{0}}\right|^{2} d x\right)^{p / 2}+1\right] \\
& =C\left(p, M_{0}\right) r_{0}^{p-p \alpha}\left[\left(\Psi\left(x_{0}, r_{0}, l_{x_{0}, r_{0}}\right)\right)^{p / 2}+1\right],
\end{aligned}
$$

where $\left|x^{\prime}-x^{\prime}{ }_{0}\right| \leq d\left(x, x_{0}\right) \leq r_{0}$ is used in the third inequality.

By the definition of $\sum_{1}$ and $\sum_{2}$, (117) and (119), we know that for any $\varepsilon_{*}$ and $\kappa_{*}$, there exists a radius $r^{\prime}: 0<r^{\prime}$ $<\min \left\{r_{*}, \operatorname{dist}\left(x_{0}, \partial \Omega\right)\right\}$ such that

$$
\begin{gathered}
\Psi\left(x_{0}, r^{\prime}, l_{x_{0}, r^{\prime}}\right)<\varepsilon_{*}, \\
Y_{\alpha}\left(x_{0}, r^{\prime}\right)<\kappa_{*} .
\end{gathered}
$$


Using the continuity of integrals, it follows that there exists a neighborhood $U \subseteq \Omega$ of $x_{0}$ so that for any $x \in U$,

$$
\begin{array}{r}
\Psi\left(x, r^{\prime}, l_{x_{0}, r^{\prime}}\right)<\varepsilon_{*}, \\
Y_{\alpha}\left(x, r^{\prime}\right)<\kappa_{*} .
\end{array}
$$

Then, Lemma 14 shows

$$
\Psi\left(x, \theta^{k} r^{\prime}, l_{x_{0}, \theta^{k} r^{\prime}}\right)<\varepsilon_{*}, Y_{\alpha}\left(x, \theta^{k} r^{\prime}\right)<\kappa_{*}, \quad \forall x \in U, k \in \mathbb{N},
$$

so

$\sup _{x \in U, r \in\left(0, r^{\prime}\right)} r^{-p \alpha} \frac{1}{\left|B_{r}(x)\right|} \int_{B_{r}(x)}\left|u-u_{x, r}\right|^{p} d y=\sup _{x \in U, r \in\left(0, r^{\prime}\right)} Y_{\alpha}(x, r)<\kappa_{*}<\infty$,

i.e., $u \in L^{p, \wp+p \alpha}\left(U, \mathbb{R}^{2 n \times N}\right)$. Therefore, we have $u \in C_{\text {loc }}^{0, \alpha}(U$ , $\mathbb{R}^{N}$ ) from Lemma 5 .

Step 2. We prove $\nabla_{H} u \in L^{p, \beta}\left(\Omega \backslash \Omega_{0}, \mathbb{R}^{2 n \times N}\right)$. For $\alpha \in(0,1)$, it implies by Lemma 7 (1) that

(1) if $\left|\nabla_{H} u-\nabla_{H} l_{x, r}\right|>1$, then $\left|\nabla_{H} u-\nabla_{H} l_{x, r}\right|^{p} \leq 2$ $\left|V\left(\nabla_{H} u-\nabla_{H} l_{x, r}\right)\right|^{2}$

(2) if $\left|\nabla_{H} u-\nabla_{H} l_{x, r}\right| \leq 1$, then $\left|\nabla_{H} u-\nabla_{H} l_{x, r}\right|^{p} \leq 1$

so

$$
\begin{aligned}
& \sup _{x \in U, r \in\left(0, r^{\prime}\right)} r^{p(1-\alpha)} \frac{1}{\left|B_{r}(x)\right|} \int_{B_{r}(x)}\left|\nabla_{H} u-\nabla_{H} l_{x, r}\right|^{p} d y \leq \sup _{x \in U, r \in\left(0, r^{\prime}\right)} r^{p(1-\alpha)} \\
& \cdot\left[\frac{2}{\left|B_{r}(x)\right|} \int_{B_{r}(x) \cap\left\{\left|\nabla_{H} u-\left(\nabla_{H} l\right)_{x, r}\right|>1\right\}}\left|V\left(\nabla_{H} u-\nabla_{H} l_{x, r}\right)\right|^{2} d y+1\right] \\
& \leq \sup _{x \in U, r \in\left(0, r^{\prime}\right)} r^{p(1-\alpha)}\left[\frac{2}{\left|B_{r}(x)\right|} \int_{B_{r}(x)}\left|V\left(\nabla_{H} u-\nabla_{H} l_{x, r}\right)\right|^{2} d y+1\right] \\
& \leq \sup _{x \in U, r \in\left(0, r^{\prime}\right)} r^{p(1-\alpha)}\left[2 C _ { c } \left(\Psi\left(x, r, l_{x, r}\right)+\omega\left(Y_{\alpha}(x, r)\right)+v(r)\right.\right. \\
& +f(x, r))+1]<\infty .
\end{aligned}
$$

Thus,

$$
\begin{aligned}
& \sup _{x \in U, r \in\left(0, r^{\prime}\right)} r^{-[\wp-p(1-\alpha)]} \int_{B_{r}(x)}\left|\nabla_{H} u\right|^{p} d y \leq C_{p} \sup _{x \in U, r \in\left(0, r^{\prime}\right)} r^{p(1-\alpha)} \\
& \cdot\left[\frac{1}{\left|B_{r}(x)\right|} \int_{B_{r}(x)}\left|\nabla_{H} u-\nabla_{H} l_{x, r}\right|^{p} d y+\left|\nabla_{H} l_{x, r}\right|^{p}\right] \\
& \quad \leq C_{p} \sup _{x \in U, r \in\left(0, r^{\prime}\right)} r^{p(1-\alpha)}\left[\frac{1}{\left|B_{r}(x)\right|} \int_{B_{r}(x)}\left|\nabla_{H} u-\nabla_{H} l_{x, r}\right|^{p} d y+M_{0}^{p}\right]<\infty,
\end{aligned}
$$

i.e., $\nabla_{H} u \in L^{p, \beta}\left(U, \mathbb{R}^{2 n \times N}\right)$, where $\beta=\wp-p(1-\alpha)$.

Therefore, Theorem 1 is proved.

\section{Data Availability}

No data is used.

\section{Conflicts of Interest}

The authors declare that they have no conflicts of interest.

\section{Acknowledgments}

This work was supported by the National Natural Science Foundation of China (No. 11771354 and No. 12061010) and the Natural Science Foundation of Jiangxi Province grant 20202BAB201004.

\section{References}

[1] J. Kohn, "Pseudo-differential operators and hypoellipticity," Proceedings of Symposia in Pure Mathematics, vol. 23, pp. 61-69, 1973.

[2] L. Hörmander, "Hypoelliptic second order differential equations," Acta Math., vol. 119, pp. 147-171, 1967.

[3] G. Folland and E. Stein, "Estimates for the $\bar{\partial}_{b}$-complex and analysis on the Heisenberg group," Communications on Pure and Applied Mathematics, vol. 27, no. 4, pp. 429522, 1974.

[4] M. Bramanti and M. Zhu, " $L^{p}$ and Schauder estimates for nonvariational operators structured on Hörmander vector fields with drift," Analysis \& PDE, vol. 6, no. 8, pp. 17931855, 2013.

[5] G. Du, J. Han, and P. Niu, "Interior regularity for degenerate equations with drift on homogeneous groups," Revista de la Real Academia de Ciencias Exactas, Físicas y Naturales. Serie A. Matemáticas, vol. 113, no. 2, pp. 587-604, 2019.

[6] X. Feng and P. Niu, "Interior regularity for degenerate elliptic equations with drift on homogeneous groups," Journal of Lie Theory, vol. 23, no. 3, pp. 803-825, 2013.

[7] Y. Hou, X. Feng, and X. Cui, "Global Hölder estimates for hypoelliptic operators with drift on homogeneous groups," Miskolc Mathematical Notes, vol. 13, no. 2, pp. 337-347, 2012.

[8] Y. Hou and P. Niu, "Weighted Sobolev-Morrey estimates for hypoelliptic operators with drift on homogeneous groups," Journal of Mathematical Analysis and Applications, vol. 428, no. 2, pp. 1319-1338, 2015.

[9] A. D. Austin and J. T. Tyson, "A new proof of the $C^{\infty}$ regularity of $C^{2}$ conformal mappings on the Heisenberg group," Colloquium Mathematicum, vol. 150, no. 2, pp. 217-228, 2017.

[10] Z. Tan and Y. Wang, "Partial regularity for subquadratic homogeneity elliptic system with VMO- coefficients," Journal of Mathematical Analysis and Applications, vol. 454, no. 2, pp. 617-638, 2017.

[11] Z. Tan, Y. Wang, and S. Chen, "Partial regularity in the interior for discontinuous inhomogeneous elliptic system with VMOcoefficients," Annali di Matematica Pura ed Applicata, vol. 196, no. 1, pp. 85-105, 2017.

[12] Z. Tan, Y. Wang, and S. Chen, "Partial regularity up to the boundary for solutions of subquadratic elliptic systems," $A d v$. Nonlinear Anal., vol. 7, no. 4, pp. 469-483, 2018.

[13] J. Wang and J. J. Manfredi, "Partial Hölder continuity for nonlinear sub-elliptic systems with VMO-coefficients in the 
Heisenberg group," Advances in Nonlinear Analysis, vol. 7, no. 1, pp. 97-116, 2018.

[14] J. Wang, Q. Liao, M. Zhu, D. Liao, and P. Hong, "Partial regularity for discontinuous sub-elliptic systems with VMO- coefficients involving controllable growth terms in Heisenberg groups," Nonlinear Analysis, vol. 178, no. 1, pp. 227-246, 2019.

[15] J. Wang, M. Zhu, S. Gao, and D. Liao, "Regularity for subelliptic systems with VMO-coefficients in the Heisenberg group: the sub-quadratic structure case," Advances in Nonlinear Analysis, vol. 10, no. 1, pp. 420-449, 2021.

[16] F. Duzaar and K. Steffen, "Optimal interior and boundary regularity for almost minimizers to elliptic variational integrals," Journal fur die Reine und Angewandte Mathematik, vol. 2002, no. 546, pp. 73-138, 2002.

[17] F. Duzaar and J. F. Grotowski, "Optimal interior partial regularity for nonlinear elliptic systems: the method of Aharmonic approximation," manuscripta mathematica, vol. 103, no. 3, pp. 267-298, 2000.

[18] F. Duzaar and G. Mingione, "The $p$-harmonic approximation and the regularity of $p$-harmonic maps," Calculus of Variations and Partial Differential Equations, vol. 20, no. 3, pp. 235-256, 2004.

[19] F. Duzaar and G. Mingione, "Regularity for degenerate elliptic problems via $p$-harmonic approximation," Annales de l'Institut Henri Poincaré C, Analyse non linéaire, vol. 21, no. 5, pp. 735-766, 2004.

[20] Y. Chen and L. Wu, Elliptic Equation and Elliptic System of Second Order(in Chinese), Science Press, Beijing, 1991.

[21] G. Lu, "Embedding theorems on Campanato-Morrey spaces for vector fields of Hörmander type," Approximation Theory and Its Applications, vol. 14, no. 1, pp. 69-80, 1998.

[22] Z. Zhou, "A generalization of Lebesgue differential theorem and its application," Acta Mathematica Scientia, vol. 21, no. 1, pp. 109-113, 2001.

[23] A. Bonfiglioli, E. Lanconelli, and F. Uguzzoni, Stratified Lie Groups and Potential Theory for Their Sub-Laplacians, Springer Monographs in Mathematics, Springer, Berlin, 2007.

[24] L. Capogna, D. Danielli, and N. Garofalo, "An embedding theorem and the Harnack inequality for nonlinear subelliptic equations," Communications in Partial Differential Equations, vol. 18, no. 9-10, pp. 1765-1794, 1993.

[25] J. Zhang and P. Niu, "Hölder regularity of quasiminimizers to generalized orlicz functional on the Heisenberg group," Journal of Function Spaces, vol. 2020, Article ID 8838654, 13 pages, 2020.

[26] M. Carozza, N. Fusco, and G. Mingione, "Partial regularity of minimizers of quasiconvex integrals with sub-quadratic growth," Annali di Matematica Pura ed Applicata, vol. 175, no. 1, pp. 141-164, 1998.

[27] Y. Ding, Foundation of Modern Analysis(in Chinese), Beijing Normal University Press, Beijing, 2008. 